\title{
LOCALIZED MORREY-CAMPANATO SPACES ON METRIC MEASURE SPACES AND APPLICATIONS TO SCHRÖDINGER OPERATORS
}

\author{
DACHUN YANG, DONGYONG YANG, AND YUAN ZHOU
}

\begin{abstract}
Let $\mathcal{X}$ be a space of homogeneous type in the sense of Coifman and Weiss, and let $\mathcal{D}$ be a collection of balls in $\mathcal{X}$. The authors introduce the localized atomic Hardy space $H_{\mathcal{D}}^{p, q}(\mathcal{X})$ with $p \in(0,1]$ and $q \in[1, \infty] \cap(p, \infty]$, the localized Morrey-Campanato space $\mathcal{E}_{\mathcal{D}}^{\alpha, p}(\mathcal{X})$, and the localized MorreyCampanato-BLO (bounded lower oscillation) space $\widetilde{\mathcal{E}}_{\mathcal{D}}^{\alpha, p}(\mathcal{X})$ with $\alpha \in \mathbb{R}$ and $p \in(0, \infty)$, and they establish their basic properties, including $H_{\mathcal{D}}^{p, q}(\mathcal{X})=$ $H_{\mathcal{D}}^{p, \infty}(\mathcal{X})$ and several equivalent characterizations for $\mathcal{E}_{\mathcal{D}}^{\alpha, p}(\mathcal{X})$ and $\widetilde{\mathcal{E}}_{\mathcal{D}}^{\alpha, p}(\mathcal{X})$. In particular, the authors prove that when $\alpha>0$ and $p \in[1, \infty)$, then $\widetilde{\mathcal{E}}_{\mathcal{D}}^{\alpha, p}(\mathcal{X})=$ $\mathcal{E}_{\mathcal{D}}^{\alpha, p}(\mathcal{X})=\operatorname{Lip}_{\mathcal{D}}(\alpha ; \mathcal{X})$, and when $p \in(0,1]$, then the dual space of $H_{\mathcal{D}}^{p, \infty}(\mathcal{X})$ is $\mathcal{E}_{\mathcal{D}}^{1 / p-1,1}(\mathcal{X})$. Let $\rho$ be an admissible function modeled on the known auxiliary function determined by the Schrödinger operator. Denote the spaces $\mathcal{E}_{\mathcal{D}}^{\alpha, p}(\mathcal{X})$ and $\widetilde{\mathcal{E}}_{\mathcal{D}}^{\alpha, p}(\mathcal{X})$, respectively, by $\mathcal{E}_{\rho}^{\alpha, p}(\mathcal{X})$ and $\widetilde{\mathcal{E}}_{\rho}^{\alpha, p}(\mathcal{X})$, when $\mathcal{D}$ is determined by $\rho$. The authors then obtain the boundedness from $\mathcal{E}_{\rho}^{\alpha, p}(\mathcal{X})$ to $\widetilde{\mathcal{E}}_{\rho}^{\alpha, p}(\mathcal{X})$ of the radial and the Poisson semigroup maximal functions and the Littlewood-Paley $g$-function, which are defined via kernels modeled on the semigroup generated by the Schrödinger operator. These results apply in a wide range of settings, for instance, the Schrödinger operator or the degenerate Schrödinger operator on $\mathbb{R}^{d}$, or the sub-Laplace Schrödinger operator on Heisenberg groups or connected and simply connected nilpotent Lie groups.
\end{abstract}

\section{$\S 1$. Introduction}

The theory of Morrey-Campanato spaces plays an important role in harmonic analysis and partial differential equations (see, e.g., [1], [24], [28], [29], $[26],[17],[22],[23],[5]$ and their references). It is well known that the dual space of the Hardy space $H^{p}\left(\mathbb{R}^{d}\right)$ with $p \in(0,1)$ is the Morrey-Campanato space $\mathcal{E}^{1 / p-1,1}\left(\mathbb{R}^{d}\right)$. Notice that Morrey-Campanato spaces on $\mathbb{R}^{d}$ are essentially related to the Laplacian $\Delta$, where $\Delta \equiv \sum_{j=1}^{d} \frac{\partial^{2}}{\partial x_{j}^{2}}$.

Received September 1, 2008. Revised October 31, 2009. Accepted November 3, 2009.

First author partially supported by National Natural Science Foundation of China grant 10871025 .

(C) 2010 by The Editorial Board of the Nagoya Mathematical Journal 
On the other hand, there exists an increasing interest in the study of Schrödinger operators on $\mathbb{R}^{d}$ and the sub-Laplace Schrödinger operators on connected and simply connected nilpotent Lie groups with nonnegative potentials satisfying the reverse Hölder inequality (see, e.g., [10], [34], [25], [18], [8], [7], [19], [33], [16]). Let $\mathcal{L} \equiv-\Delta+V$ be the Schrödinger operator on $\mathbb{R}^{d}$, where the potential $V$ is a nonnegative locally integrable function. Denote by $\mathcal{B}_{q}\left(\mathbb{R}^{d}\right)$ the class of functions satisfying the reverse Hölder inequality of order $q$. For $V \in \mathcal{B}_{d / 2}\left(\mathbb{R}^{d}\right)$ with $d \geq 3$, Dziubański et al. ([8], [9], [7]) studied the BMO (bounded mean oscillation)-type space $\mathrm{BMO}_{\mathcal{L}}\left(\mathbb{R}^{d}\right)$ and the Hardy space $H_{\mathcal{L}}^{p}\left(\mathbb{R}^{d}\right)$ with $p \in(d /(d+1), 1]$ and, especially, proved that the dual space of $H_{\mathcal{L}}^{1}\left(\mathbb{R}^{d}\right)$ is $\operatorname{BMO}_{\mathcal{L}}\left(\mathbb{R}^{d}\right)$. Moreover, they obtained the boundedness on these spaces of the variants of several classical operators, including the radial maximal function and the Littlewood-Paley $g$-function associated to $\mathcal{L}$. Recently, Huang and Liu [16] further proved that the dual space of $H_{\mathcal{L}}^{p}\left(\mathbb{R}^{d}\right)$ is certain Morrey-Campanato space. Let $\mathcal{X}$ be an $\mathrm{RD}$ (reverse doubling)-space in [12], which means that $\mathcal{X}$ is a space of homogeneous type in the sense of Coifman and Weiss $([3],[4])$ with the additional property that a reverse doubling condition holds. Let $\rho$ be a given admissible function modeled on the known auxiliary function determined by $V \in \mathcal{B}_{d / 2}\left(\mathbb{R}^{d}\right)$ (see [33] or (2.3) below). Then the localized Hardy space $H_{\rho}^{1}(\mathcal{X})$, the BMO-type space $\mathrm{BMO}_{\rho}(\mathcal{X})$, and the BLO-type space $\mathrm{BLO}_{\rho}(\mathcal{X})$ were introduced and studied by the authors of this article in [33] and [32]. Moreover, the boundedness from $\mathrm{BMO}_{\rho}(\mathcal{X})$ to $\mathrm{BLO}_{\rho}(\mathcal{X})$ of several maximal operators and the Littlewood-Paley $g$-function, which are defined via kernels modeled on the semigroup generated by the Schrödinger operator, was obtained in [32].

The first purpose of this article is to investigate behaviors of these operators on localized Morrey-Campanato spaces on metric measure spaces. To be precise, let $\mathcal{X}$ be a space of homogeneous type, which is not necessary to be an RD-space, and let $\mathcal{D}$ be a collection of balls in $\mathcal{X}$. In Section 2, we first introduce the localized atomic Hardy space $H_{\mathcal{D}}^{p, q}(\mathcal{X})$ with $p \in(0,1]$ and $q \in[1, \infty] \cap(p, \infty]$, the localized Morrey-Campanato space $\mathcal{E}_{\mathcal{D}}^{\alpha, p}(\mathcal{X})$, and the localized Morrey-Campanato-BLO space $\widetilde{\mathcal{E}}_{\mathcal{D}}^{\alpha, p}(\mathcal{X})$ with $\alpha \in \mathbb{R}$ and $p \in(0, \infty)$, and we establish their basic properties, including $H_{\mathcal{D}}^{p, q}(\mathcal{X})=H_{\mathcal{D}}^{p, \infty}(\mathcal{X})$ and several equivalent characterizations for $\mathcal{E}_{\mathcal{D}}^{\alpha, p}(\mathcal{X})$ and $\widetilde{\mathcal{E}}_{\mathcal{D}}^{\alpha, p}(\mathcal{X})$. Especially, we prove that when $\alpha>0$ and $p \in[1, \infty)$, then $\widetilde{\mathcal{E}}_{\mathcal{D}}^{\alpha, p}(\mathcal{X})=\mathcal{E}_{\mathcal{D}}^{\alpha, p}(\mathcal{X})=\operatorname{Lip}_{\mathcal{D}}(\alpha ; \mathcal{X})$, and when $p \in(0,1]$, then the dual space of 
$H_{\mathcal{D}}^{p, \infty}(\mathcal{X})$ is $\mathcal{E}_{\mathcal{D}}^{1 / p-1,1}(\mathcal{X})$ (see Theorem 2.1 below). Let $\rho$ be a given admissible function. Modeled on the semigroup generated by the Schrödinger operator, in Sections 3 and 4 we introduced the radial maximal operators $T^{+}$and $P^{+}$and Littlewood-Paley $g$-function $g(\cdot)$. Then we establish the boundedness of $T^{+}$and $P^{+}$from $\mathcal{E}_{\rho}^{\alpha, p}(\mathcal{X})$ to $\widetilde{\mathcal{E}}_{\rho}^{\alpha, p}(\mathcal{X})$ (see Theorems 3.1 and 3.2 below). Here, for the set $\mathcal{D}$ determined by $\rho$, we denote $\mathcal{E}_{\mathcal{D}}^{\alpha, p}(\mathcal{X})$ and $\widetilde{\mathcal{E}}_{\mathcal{D}}^{\alpha, p}(\mathcal{X})$, respectively, by $\mathcal{E}_{\rho}^{\alpha, p}(\mathcal{X})$ and $\widetilde{\mathcal{E}}_{\rho}^{\alpha, p}(\mathcal{X})$. Moreover, under the assumption that $g$-function $g(\cdot)$ is bounded on $L^{p}(\mathcal{X})$ with $p \in(1, \infty)$, we prove that for every $f \in \mathcal{E}_{\rho}^{\alpha, p}(\mathcal{X}),[g(f)]^{2} \in \widetilde{\mathcal{E}}_{\rho}^{2 \alpha, p / 2}(\mathcal{X})$ with norm no more than $C\|f\|_{\mathcal{E}_{\rho}^{\alpha, p}(\mathcal{X})}^{2}$, where $C$ is a positive constant independent of $f$ (see Theorem 4.1 below). As a simple corollary of this, we obtain the boundedness of $g(\cdot)$ from $\mathcal{E}_{\rho}^{\alpha, p}(\mathcal{X})$ to $\widetilde{\mathcal{E}}_{\rho}^{\alpha, p}(\mathcal{X})$. Notice that $\mathcal{E}_{\rho}^{0, p}(\mathcal{X})=\operatorname{BMO}_{\rho}(\mathcal{X})$ and that $\widetilde{\mathcal{E}}_{\rho}^{0, p}(\mathcal{X})=\mathrm{BLO}_{\rho}(\mathcal{X})$ when $p \in[1, \infty)$. Thus, the results in Sections 3 and 4 when $\alpha=0$ and $\mathcal{X}$ is an RD-space were already obtained in [32].

Finally, as the second purpose of this article, in Section 5 we apply results obtained in Sections 3 and 4, respectively, to the Schrödinger operator or the degenerate Schrödinger operator on $\mathbb{R}^{d}$ and to the sub-Laplace Schrödinger operator on Heisenberg groups or on connected and simply connected nilpotent Lie groups (see Propositions 5.1-5.5 below). The nonnegative potentials of these Schrödinger operators are assumed to satisfy the reverse Hölder inequality.

We now state some conventions. Throughout this article, we always use $C$ to denote a positive constant that is independent of the main parameters involved but whose value may differ from line to line. Constants with subscripts, such as $C_{1}$ and $A_{1}$, do not change in different occurrences. If $f \leq C g$, we then write $f \lesssim g$ or $g \gtrsim f$, and if $f \lesssim g \lesssim f$, we then write $f \sim g$. For any given "normed" spaces $\mathcal{A}$ and $\mathcal{B}$, the symbol $\mathcal{A} \subset \mathcal{B}$ means that, for all $f \in \mathcal{A}, f \in \mathcal{B}$ and $\|f\|_{\mathcal{B}} \lesssim\|f\|_{\mathcal{A}}$. We also use $B$ to denote a ball of $\mathcal{X}$, and for $\lambda>0, \lambda B$ denotes the ball with the same center as $B$ but radius $\lambda$ times the radius of $B$. Moreover, set $B^{\complement} \equiv \mathcal{X} \backslash B$. Also, for any set $E \subset \mathcal{X}$, $\chi_{E}$ denotes its characteristic function. For all $f \in L_{\text {loc }}^{1}(\mathcal{X})$ and balls $B$, we always set $f_{B} \equiv 1 /(\mu(B)) \int_{B} f(y) d \mu(y)$.

\section{§2. Localized Morrey-Campanato and Hardy spaces}

This section is divided into two subsections. In Section 2.1, we introduce the localized spaces $\mathcal{E}_{\mathcal{D}}^{\alpha, p}(\mathcal{X})$ and $\widetilde{\mathcal{E}}_{\mathcal{D}}^{\alpha, p}(\mathcal{X})$ with $\alpha \in \mathbb{R}$ and $p \in(0, \infty)$; we then establish the relations of these localized spaces with their corresponding 
global versions and prove that, for all $\alpha \in[0, \infty)$ and $p \in(1, \infty), \mathcal{E}_{\mathcal{D}}^{\alpha, p}(\mathcal{X})=$ $\mathcal{E}_{\mathcal{D}}^{\alpha, 1}(\mathcal{X})$ and $\widetilde{\mathcal{E}}_{\mathcal{D}}^{\alpha, p}(\mathcal{X})=\widetilde{\mathcal{E}}_{\mathcal{D}}^{\alpha, 1}(\mathcal{X})$. In Section 2.2 , we introduce the localized space $H_{\mathcal{D}}^{p, q}(\mathcal{X})$ with $p \in(0,1]$ and $q \in[1, \infty] \cap(p, \infty]$, and we show that $H_{\mathcal{D}}^{p, q}(\mathcal{X})=H_{\mathcal{D}}^{p, \infty}(\mathcal{X})$ and that the dual space of $H_{\mathcal{D}}^{p, \infty}(\mathcal{X})$ is $\mathcal{E}_{\mathcal{D}}^{1 / p-1,1}(\mathcal{X})$

\subsection{Localized Morrey-Campanato spaces}

We first recall the notion of spaces of homogeneous type in [3] and [4].

Definition 2.1. Let $(\mathcal{X}, d)$ be a metric space endowed with a regular Borel measure $\mu$ such that all balls defined by $d$ have finite and positive measure. For any $x \in \mathcal{X}$ and $r \in(0, \infty)$, set the ball $B(x, r) \equiv\{y \in \mathcal{X}$ : $d(x, y)<r\}$. The triple $(\mathcal{X}, d, \mu)$ is called a space of homogeneous type if there exists a constant $A_{1} \in[1, \infty)$ such that for all $x \in \mathcal{X}$ and $r \in(0, \infty)$,

$$
\mu(B(x, 2 r)) \leq A_{1} \mu(B(x, r)) \quad \text { (doubling property) }
$$

From (2.1), it is not difficult to see that there exist positive constants $A_{2}$ and $n$ such that for all $x \in \mathcal{X}, r \in(0, \infty)$, and $\lambda \in[1, \infty)$,

$$
\mu(B(x, \lambda r)) \leq A_{2} \lambda^{n} \mu(B(x, r)) .
$$

In what follows, we always set $V_{r}(x) \equiv \mu(B(x, r))$ and $V(x, y) \equiv \mu(B(x$, $d(x, y)))$ for all $x, y \in \mathcal{X}$ and $r \in(0, \infty)$.

Definition 2.2. ([33]) A positive function $\rho$ on $\mathcal{X}$ is said to be admissible if there exist positive constants $C_{0}$ and $k_{0}$ such that for all $x, y \in \mathcal{X}$,

$$
\frac{1}{\rho(x)} \leq C_{0} \frac{1}{\rho(y)}\left(1+\frac{d(x, y)}{\rho(y)}\right)^{k_{0}} .
$$

Obviously, if $\rho$ is a constant function, then $\rho$ is admissible. Moreover, let $x_{0} \in \mathcal{X}$ be fixed. The function $\rho(y) \equiv\left(1+d\left(x_{0}, y\right)\right)^{s}$ for all $y \in \mathcal{X}$ with $s \in(-\infty, 1)$ also satisfies Definition 2.2 with $k_{0}=s /(1-s)$ when $s \in[0,1)$ and with $k_{0}=-s$ when $s \in(-\infty, 0)$. Another nontrivial class of admissible functions is given by the well-known reverse Hölder class $\mathcal{B}_{q}(\mathcal{X}, d, \mu)$, which is written as $\mathcal{B}_{q}(\mathcal{X})$ for simplicity. Recall that a nonnegative potential $V$ is said to be in $\mathcal{B}_{q}(\mathcal{X})$ with $q \in(1, \infty]$ if there exists a positive constant $C$ such that for all balls $B$ of $\mathcal{X}$,

$$
\left(\frac{1}{|B|} \int_{B}[V(y)]^{q} d \mu(y)\right)^{1 / q} \leq \frac{C}{|B|} \int_{B} V(y) d \mu(y)
$$


with the usual modification made when $q=\infty$. It is known that, if $V \in$ $\mathcal{B}_{q}(\mathcal{X})$ for certain $q \in(1, \infty]$ and $V(y) d \mu(y)$ is doubling then, $V$ is an $A_{\infty}(\mathcal{X})$ weight in the sense of Muckenhoupt, and also $V \in \mathcal{B}_{q+\epsilon}(\mathcal{X})$ for some $\epsilon \epsilon$ $(0, \infty)$ (see, e.g., [26], [27]). Thus $\mathcal{B}_{q}(\mathcal{X})=\bigcup_{q_{1}>q} \mathcal{B}_{q_{1}}(\mathcal{X})$. For all $V \in \mathcal{B}_{q}(\mathcal{X})$ with certain $q \in(1, \infty]$ and for all $x \in \mathcal{X}$, set

$$
\rho(x) \equiv[m(x, V)]^{-1} \equiv \sup \left\{r>0: \frac{r^{2}}{\mu(B(x, r))} \int_{B(x, r)} V(y) d \mu(y) \leq 1\right\}
$$

(see, e.g., [25], [33]). It was also proved in [33] that $\rho$ in (2.3) is an admissible function if $q>\max \{1, n / 2\}, V \in \mathcal{B}_{q}(\mathcal{X})$ and $V(y) d \mu(y)$ is doubling.

We now recall the notion of Morrey-Campanato spaces and introduce the definitions of Morrey-Campanato-BLO spaces and their localized versions.

Definition 2.3. Let $\alpha \in \mathbb{R}$, and let $p \in(0, \infty)$.

(i) A function $f \in L_{\text {loc }}^{p}(\mathcal{X})$ is said to be in the Morrey-Campanato space $\mathcal{E}^{\alpha, p}(\mathcal{X})$ if

$$
\|f\|_{\mathcal{E}^{\alpha, p}(\mathcal{X})} \equiv \sup _{B \subset \mathcal{X}}\left\{\frac{1}{[\mu(B)]^{1+p \alpha}} \int_{B}\left|f(y)-f_{B}\right|^{p} d \mu(y)\right\}^{1 / p}<\infty,
$$

where the supremum is taken over all balls $B \subset \mathcal{X}$ and $f_{B}=1 /(\mu(B)) \times$ $\int_{B} f(z) d \mu(z)$.

(ii) A function $f \in L_{\text {loc }}^{p}(\mathcal{X})$ is said to be in the Morrey-Campanato-BLO space $\widetilde{\mathcal{E}}^{\alpha, p}(\mathcal{X})$ if

$$
\|f\|_{\widetilde{\mathcal{E}}^{\alpha, p}(\mathcal{X})} \equiv \sup _{B \subset \mathcal{X}}\left\{\frac{1}{[\mu(B)]^{1+p \alpha}} \int_{B}[f(y)-\underset{B}{\operatorname{essinf}} f]^{p} d \mu(y)\right\}^{1 / p}<\infty,
$$

where the supremum is taken over all balls $B \subset \mathcal{X}$.

(iii) Let $\alpha \in(0, \infty)$. A function $f$ on $\mathcal{X}$ is said to be in the Lipschitz space $\operatorname{Lip}(\alpha ; \mathcal{X})$ if there exists a nonnegative constant $C$ such that for all $x, y \in \mathcal{X}$ and balls $B$ containing $x$ and $y$,

$$
|f(x)-f(y)| \leq C[\mu(B)]^{\alpha} .
$$

The minimal nonnegative constant $C$ as above is called the norm of $f$ in $\operatorname{Lip}(\alpha ; \mathcal{X})$ and is denoted by $\|f\|_{\operatorname{Lip}(\alpha ; \mathcal{X})}$.

REMARK 2.1. (i) The space $\mathcal{E}^{\alpha, p}(\mathcal{X})$ was first introduced by Campanato [1] when $\mathcal{X}$ is a bounded subset of $\mathbb{R}^{d}$ and $\mu$ is the $d$-dimensional Lebesgue 
measure. When $\alpha=0, \mathcal{E}^{0, p}(\mathcal{X})$ is just the space $\operatorname{BMO}^{p}(\mathcal{X})$, and $\mathcal{E}^{0, p}(\mathcal{X})$ with $p \in[1, \infty)$ coincides with $\mathrm{BMO}^{1}(\mathcal{X})$ (see [4]). For simplicity, we denote $\operatorname{BMO}^{1}(\mathcal{X})$ by $\operatorname{BMO}(\mathcal{X})$.

(ii) The space $\widetilde{\mathcal{E}}^{0, p}(\mathcal{X})$ is just the space $\mathrm{BLO}^{p}(\mathcal{X})$. By (i) of this remark and the fact that $\mathrm{BLO}^{1}(\mathcal{X}) \subset \operatorname{BMO}(\mathcal{X})$, it is easy to see that $\widetilde{\mathcal{E}}^{0, p}(\mathcal{X})$ with $p \in[1, \infty)$ coincides with $\mathrm{BLO}^{1}(\mathcal{X})$. For simplicity, we denote $\mathrm{BLO}^{1}(\mathcal{X})$ by $\operatorname{BLO}(\mathcal{X})$. Recall that $\operatorname{BLO}(\mathcal{X})$ and $\widetilde{\mathcal{E}}^{\alpha, p}(\mathcal{X})$ are not linear spaces. The space $\operatorname{BLO}\left(\mathbb{R}^{d}\right)$ was first introduced by Coifman and Rochberg [2], and $\widetilde{\mathcal{E}}^{\alpha, p}\left(\mathbb{R}^{d}\right)$ was introduced in [14].

(iii) When $\alpha \in \mathbb{R}$ and $p \in[1, \infty), \widetilde{\mathcal{E}}^{\alpha, p}(\mathcal{X}) \subset \mathcal{E}^{\alpha, p}(\mathcal{X})$. Moreover, when $\alpha \in$ $(0, \infty)$ and $p \in[1, \infty)$, we have $\widetilde{\mathcal{E}}^{\alpha, p}(\mathcal{X})=\mathcal{E}^{\alpha, p}(\mathcal{X})=\operatorname{Lip}(\alpha ; \mathcal{X})$ with equivalent norms. In fact, Macías and Segovia [20] proved that when $\alpha \in(0, \infty)$ and $p \in[1, \infty), \mathcal{E}^{\alpha, p}(\mathcal{X})=\operatorname{Lip}(\alpha ; \mathcal{X})$. On the other hand, for all $f \in \mathcal{E}^{\alpha, p}(\mathcal{X})$ and balls $B$,

$$
\begin{aligned}
\int_{B}\left[f(y)-\underset{B}{\operatorname{essinf} f]^{p}} d \mu(y)\right. & \leq \int_{B} \operatorname{esssup}_{x \in B}|f(y)-f(x)|^{p} d \mu(y) \\
& \lesssim\|f\|_{\operatorname{Lip}(\alpha ; \mathcal{X})}^{p}[\mu(B)]^{1+p \alpha}
\end{aligned}
$$

which implies that $\|f\|_{\widetilde{\mathcal{E}}^{\alpha, p}(\mathcal{X})} \lesssim\|f\|_{\operatorname{Lip}(\alpha ; \mathcal{X})} \sim\|f\|_{\mathcal{E}^{\alpha, p}(\mathcal{X})}$. Thus, $\mathcal{E}^{\alpha, p}(\mathcal{X}) \subset$ $\widetilde{\mathcal{E}}^{\alpha, p}(\mathcal{X})$, and the claim holds.

Definition 2.4. Let $\mathcal{D}$ be a collection of balls in $\mathcal{X}$, let $p \in(0, \infty)$, and let $\alpha \in \mathbb{R}$. Denote by $B$ any ball of $\mathcal{X}$.

(i) A function $f \in L_{\text {loc }}^{p}(\mathcal{X})$ is said to be in the localized Morrey-Campanato space $\mathcal{E}_{\mathcal{D}}^{\alpha, p}(\mathcal{X})$ if

$$
\begin{aligned}
\|f\|_{\mathcal{E}_{\mathcal{D}}^{\alpha, p}(\mathcal{X})} \equiv & \sup _{B \notin \mathcal{D}}\left\{\frac{1}{[\mu(B)]^{1+p \alpha}} \int_{B}\left|f(y)-f_{B}\right|^{p} d \mu(y)\right\}^{1 / p} \\
& +\sup _{B \in \mathcal{D}}\left\{\frac{1}{[\mu(B)]^{1+p \alpha}} \int_{B}|f(y)|^{p} d \mu(y)\right\}^{1 / p}<\infty
\end{aligned}
$$

where $f_{B}=1 /(\mu(B)) \int_{B} f(z) d \mu(z)$.

(ii) A function $f \in L_{\text {loc }}^{p}(\mathcal{X})$ is said to be in the localized Morrey-Campanato$B L O$ space $\widetilde{\mathcal{E}}_{\mathcal{D}}^{\alpha, p}(\mathcal{X})$ if

$$
\|f\|_{\widetilde{\mathcal{E}}_{\mathcal{D}}^{\alpha, p}(\mathcal{X})} \equiv \sup _{B \notin \mathcal{D}}\left\{\frac{1}{[\mu(B)]^{1+p \alpha}} \int_{B}[f(y)-\underset{B}{\operatorname{essinf} f}]^{p} d \mu(y)\right\}^{1 / p}
$$




$$
+\sup _{B \in \mathcal{D}}\left\{\frac{1}{[\mu(B)]^{1+p \alpha}} \int_{B}|f(y)|^{p} d \mu(y)\right\}^{1 / p}<\infty .
$$

(iii) Let $\alpha \in(0, \infty)$. A function $f$ on $\mathcal{X}$ is said to be in the localized Lipschitz space $\operatorname{Lip}_{\mathcal{D}}(\alpha ; \mathcal{X})$ if there exists a nonnegative constant $C$ such that for all $x, y \in \mathcal{X}$ and balls $B$ containing $x$ and $y$ with $B \notin \mathcal{D}$,

$$
|f(x)-f(y)| \leq C[\mu(B)]^{\alpha},
$$

and that, for all balls $B \in \mathcal{D},\|f\|_{L^{\infty}(B)} \leq C[\mu(B)]^{\alpha}$. The minimal nonnegative constant $C$ as above is called the norm of $f$ in $\operatorname{Lip}_{\mathcal{D}}(\alpha ; \mathcal{X})$ and is denoted by $\|f\|_{\operatorname{Lip}_{\mathcal{D}}(\alpha ; \mathcal{X})}$.

REMARK 2.2. (i) When $\alpha=0$ and $p \in[1, \infty)$, we denote $\mathcal{E}_{\mathcal{D}}^{0, p}(\mathcal{X})$ by $\mathrm{BMO}_{\mathcal{D}}^{p}(\mathcal{X})$, and we denote $\mathrm{BMO}_{\mathcal{D}}^{1}(\mathcal{X})$ by $\mathrm{BMO}_{\mathcal{D}}(\mathcal{X})$. We also denote $\widetilde{\mathcal{E}}_{\mathcal{D}}^{0, p}(\mathcal{X})$ by $\operatorname{BLO}_{\mathcal{D}}^{p}(\mathcal{X})$, and we denote $\widetilde{\mathcal{E}}_{\mathcal{D}}^{0,1}(\mathcal{X})$ by $\mathrm{BLO}_{\mathcal{D}}(\mathcal{X})$. The localized BLO space was first introduced in [15] in the setting of $\mathbb{R}^{d}$ endowed with a nondoubling measure.

(ii) If $\mathcal{X}$ is the Euclidean space $\mathbb{R}^{d}$ and $\mathcal{D} \equiv\left\{B(x, r): r \geq 1, x \in \mathbb{R}^{d}\right\}$, then $\mathrm{BMO}_{\mathcal{D}}(\mathcal{X})$ is just the localized BMO space of Goldberg [11], and $\operatorname{Lip}_{\mathcal{D}}(\alpha ; \mathcal{X})$ with $\alpha \in(0,1)$ is just the inhomogeneous Lipschitz space (see also [11]).

(iii) For all $\alpha \in \mathbb{R}$ and $p \in(0, \infty), \widetilde{\mathcal{E}}_{\mathcal{D}}^{\alpha, p}(\mathcal{X}) \subset \mathcal{E}_{\mathcal{D}}^{\alpha, p}(\mathcal{X}) \subset \mathcal{E}^{\alpha, p}(\mathcal{X})$. For $\alpha \in$ $(0, \infty), \operatorname{Lip}_{\mathcal{D}}(\alpha ; \mathcal{X}) \subset \operatorname{Lip}(\alpha ; \mathcal{X})$.

(iv) Let $\rho$ be an admissible function, and let $\mathcal{D}_{\rho} \equiv\{B(x, r): x \in \mathcal{X}, r \geq$ $\rho(x)\}$. In this case, we denote $\mathcal{E}_{\mathcal{D}_{\rho}}^{\alpha, p}(\mathcal{X}), \widetilde{\mathcal{E}}_{\mathcal{D}_{\rho}}^{\alpha, p}(\mathcal{X}), \operatorname{Lip}_{\mathcal{D}_{\rho}}(\alpha ; \mathcal{X}), \operatorname{BMO}_{\mathcal{D}_{\rho}}(\mathcal{X})$, and $\mathrm{BLO}_{\mathcal{D}_{\rho}}(\mathcal{X})$, respectively, by $\mathcal{E}_{\rho}^{\alpha, p}(\mathcal{X}), \widetilde{\mathcal{E}}_{\rho}^{\alpha, p}(\mathcal{X}), \operatorname{Lip}_{\rho}(\alpha ; \mathcal{X}), \operatorname{BMO}_{\rho}(\mathcal{X})$, and $\mathrm{BLO}_{\rho}(\mathcal{X})$. In [32], the spaces $\mathrm{BMO}_{\rho}(\mathcal{X})$ and $\mathrm{BLO}_{\rho}(\mathcal{X})$ when $\mathcal{X}$ is an RD-space were introduced.

The following results follow from Definitions 2.3 and 2.4.

Lemma 2.1. Let $\mathcal{D}$ be a collection of balls in $\mathcal{X}, p \in[1, \infty)$ and $\alpha \in \mathbb{R}$.

(i) Then $f \in \mathcal{E}_{\mathcal{D}}^{\alpha, p}(\mathcal{X})$ if and only if $f \in \mathcal{E}^{\alpha, p}(\mathcal{X})$ and $\sup _{B \in \mathcal{D}}\left|f_{B}\right| \times$ $[\mu(B)]^{-\alpha}<\infty ;$ moreover

$$
\|f\|_{\mathcal{E}_{\mathcal{D}}^{\alpha, p}(\mathcal{X})} \sim\|f\|_{\mathcal{E}^{\alpha, p}(\mathcal{X})}+\sup _{B \in \mathcal{D}}\left|f_{B}\right|[\mu(B)]^{-\alpha} .
$$

(ii) Then $f \in \widetilde{\mathcal{E}}_{\mathcal{D}}^{\alpha, p}(\mathcal{X})$ if and only if $f \in \widetilde{\mathcal{E}}^{\alpha, p}(\mathcal{X})$ and $\sup _{B \in \mathcal{D}}\left|f_{B}\right| \times$ $[\mu(B)]^{-\alpha}<\infty ;$ moreover

$$
\|f\|_{\widetilde{\mathcal{E}}_{\mathcal{D}}^{\alpha, p}(\mathcal{X})} \sim\|f\|_{\widetilde{\mathcal{E}}^{\alpha, p}(\mathcal{X})}+\sup _{B \in \mathcal{D}}\left|f_{B}\right|[\mu(B)]^{-\alpha} .
$$


(iii) Let $\alpha \in(0, \infty)$. Then $f \in \operatorname{Lip}_{\mathcal{D}}(\alpha ; \mathcal{X})$ if and only if $f \in \operatorname{Lip}(\alpha ; \mathcal{X})$ and $\sup _{B \in \mathcal{D}}[\mu(B)]^{-\alpha}\|f\|_{L^{\infty}(B)}<\infty$ or $\sup _{B \in \mathcal{D}}\left|f_{B}\right|[\mu(B)]^{-\alpha}<\infty$; moreover,

$$
\begin{aligned}
\|f\|_{\operatorname{Lip}_{\mathcal{D}}(\alpha ; \mathcal{X})} & \sim\|f\|_{\operatorname{Lip}(\alpha ; \mathcal{X})}+\sup _{B \in \mathcal{D}}\|f\|_{L^{\infty}(B)}[\mu(B)]^{-\alpha} \\
& \sim\|f\|_{\operatorname{Lip}(\alpha ; \mathcal{X})}+\sup _{B \in \mathcal{D}}\left|f_{B}\right|[\mu(B)]^{-\alpha}
\end{aligned}
$$

Proof. We first prove (i). If $f \in \mathcal{E}^{\alpha, p}(\mathcal{X})$ and $\sup _{B \in \mathcal{D}}\left|f_{B}\right|[\mu(B)]^{-\alpha}<\infty$, from Definitions 2.3 and 2.4 , it follows that

$$
\|f\|_{\mathcal{E}_{\mathcal{D}}^{\alpha, p}(\mathcal{X})} \leq 2\|f\|_{\mathcal{E}^{\alpha, p}(\mathcal{X})}+\sup _{B \in \mathcal{D}}\left|f_{B}\right|[\mu(B)]^{-\alpha} .
$$

Conversely, if $f \in \mathcal{E}_{\mathcal{D}}^{\alpha, p}(\mathcal{X})$, then by the Hölder inequality we have

$$
\begin{aligned}
\|f\|_{\mathcal{E}^{\alpha, p}(\mathcal{X})}+\sup _{B \in \mathcal{D}}\left|f_{B}\right|[\mu(B)]^{-\alpha} & \leq\|f\|_{\mathcal{E}_{\mathcal{D}}^{\alpha, p}(\mathcal{X})}+2 \sup _{B \in \mathcal{D}}\left|f_{B}\right|[\mu(B)]^{-\alpha} \\
& \leq 3\|f\|_{\mathcal{E}_{\mathcal{D}}^{\alpha, p}(\mathcal{X})}
\end{aligned}
$$

which together with (2.4) gives (i).

The proofs of (ii) and (iii) are similar. We omit the details, which completes the proof of Lemma 2.1 .

Lemma 2.2. Let $\mathcal{D}$ be a collection of balls in $\mathcal{X}$, and let $p \in[1, \infty)$.

(i) Then $\mathrm{BMO}_{\mathcal{D}}(\mathcal{X})=\mathrm{BMO}_{\mathcal{D}}^{p}(\mathcal{X})$ and $\mathrm{BLO}_{\mathcal{D}}(\mathcal{X})=\mathrm{BLO}_{\mathcal{D}}^{p}(\mathcal{X})$ with equivalent norms.

(ii) When $\alpha \in(0, \infty), \widetilde{\mathcal{E}}_{\mathcal{D}}^{\alpha, p}(\mathcal{X})=\mathcal{E}_{\mathcal{D}}^{\alpha, p}(\mathcal{X})=\operatorname{Lip}_{\mathcal{D}}(\alpha ; \mathcal{X})$ with equivalent norms.

Proof. To prove (i), we first assume that $f \in \mathrm{BMO}_{\mathcal{D}}^{p}(\mathcal{X})$. Then by the Hölder inequality, we have $f \in \mathrm{BMO}_{\mathcal{D}}(\mathcal{X})$ and $\|f\|_{\mathrm{BMO}_{\mathcal{D}}(\mathcal{X})} \leq\|f\|_{\mathrm{BMO}_{\mathcal{D}}^{p}(\mathcal{X})}$. Conversely, if $f \in \mathrm{BMO}_{\mathcal{D}}(\mathcal{X})$, then from Lemma 2.1(i), with $\alpha=0$, and Remarks 2.1(i) and 2.2(iii), it follows that

$$
\|f\|_{\mathrm{BMO}_{\mathcal{D}}^{p}(\mathcal{X})} \lesssim\|f\|_{\mathrm{BMO}^{p}(\mathcal{X})}+\sup _{B \in \mathcal{D}}\left|f_{B}\right| \lesssim\|f\|_{\mathrm{BMO}_{\mathcal{D}}(\mathcal{X})}
$$

which implies that $f \in \mathrm{BMO}_{\mathcal{D}}^{p}(\mathcal{X})$ and $\|f\|_{\mathrm{BMO}_{\mathcal{D}}^{p}(\mathcal{X})} \lesssim\|f\|_{\mathrm{BMO}_{\mathcal{D}}(\mathcal{X})}$. Thus $\mathrm{BMO}_{\mathcal{D}}(\mathcal{X})=\mathrm{BMO}_{\mathcal{D}}^{p}(\mathcal{X})$ with equivalent norms. The proof for $\mathrm{BLO}_{\mathcal{D}}(\mathcal{X})=$ $\mathrm{BLO}_{\mathcal{D}}^{p}(\mathcal{X})$ is similar, and we omit the details.

To prove (ii), by Lemma 2.1 and Remark 2.1(iii), we obtain

$$
\|f\|_{\mathcal{E}_{\mathcal{D}}^{\alpha, p}(\mathcal{X})} \sim\|f\|_{\mathcal{E}^{\alpha, p}(\mathcal{X})}+\sup _{B \in \mathcal{D}}\left|f_{B}\right|[\mu(B)]^{-\alpha}
$$




$$
\begin{aligned}
& \text { LOCALized MorRey-CAMPANATO SPACES } \\
& \sim\|f\|_{\widetilde{\mathcal{E}}^{\alpha, p}(\mathcal{X})}+\sup _{B \in \mathcal{D}}\left|f_{B}\right|[\mu(B)]^{-\alpha} \sim\|f\|_{\widetilde{\mathcal{E}}_{\mathcal{D}}^{\alpha, p}(\mathcal{X})} \\
& \sim\|f\|_{\operatorname{Lip}(\alpha ; \mathcal{X})}+\sup _{B \in \mathcal{D}}\left|f_{B}\right|[\mu(B)]^{-\alpha} \sim\|f\|_{\operatorname{Lip}_{\mathcal{D}}(\alpha ; \mathcal{X})},
\end{aligned}
$$

which implies (ii). This finishes the proof of Lemma 2.2.

The space $\mathcal{X}$ is said to have the reverse doubling property if there exist constants $\kappa \in(0, n]$ and $A_{3} \in(0,1]$ such that, for all $x \in \mathcal{X}, r \in(0, \operatorname{diam}(\mathcal{X}) / 2]$, and $\lambda \in[1, \operatorname{diam}(\mathcal{X}) /(2 r)]$,

$$
A_{3} \lambda^{\kappa} \mu(B(x, r)) \leq \mu(B(x, \lambda r)) .
$$

If $(\mathcal{X}, d, \mu)$ satisfies conditions $(2.1)$ and $(2.5)$, then $(\mathcal{X}, d, \mu)$ is called an $R D$-space (see [12]).

Lemma 2.3. Let $\mathcal{X}$ be an RD-space, let $\rho$ be an admissible function on $\mathcal{X}$, and let $\mathcal{D}_{\rho}$ be as in Remark 2.2(iv). If $\alpha \in(-\infty, 0)$ and $p \in[1, \infty)$, then

$$
\|f\|_{\mathcal{E}_{\rho}^{\alpha, p}(\mathcal{X})} \sim \sup _{B \subset \mathcal{X}}\left\{\frac{1}{[\mu(B)]^{1+\alpha p}} \int_{B}|f(x)|^{p} d \mu(x)\right\}^{1 / p} .
$$

Proof. An application of the Hölder inequality leads to

$$
\|f\|_{\mathcal{E}_{\rho}^{\alpha, p}(\mathcal{X})} \lesssim \sup _{B \subset \mathcal{X}}\left\{\frac{1}{[\mu(B)]^{1+\alpha p}} \int_{B}|f(x)|^{p} d \mu(x)\right\}^{1 / p} .
$$

Conversely, if $B \in \mathcal{D}_{\rho}$, then by Definition 2.4(i) we have

$$
\left\{\frac{1}{[\mu(B)]^{1+\alpha p}} \int_{B}|f(x)|^{p} d \mu(x)\right\}^{1 / p} \leq\|f\|_{\mathcal{E}_{\rho}^{\alpha, p}(\mathcal{X})} .
$$

Now we assume that $B \equiv B\left(x_{0}, r\right) \notin \mathcal{D}_{\rho}$. Let $J_{0} \in \mathbb{N}$ such that $2^{J_{0}-1} r<$ $\rho\left(x_{0}\right) \leq 2^{J_{0}} r$. From $\alpha \in(-\infty, 0),(2.1),(2.5)$, and the Hölder inequality, it follows that

$$
\begin{aligned}
& \left\{\frac{1}{[\mu(B)]^{1+\alpha p}} \int_{B}|f(x)|^{p} d \mu(x)\right\}^{1 / p} \\
& \leq \frac{1}{[\mu(B)]^{\alpha}}\left\{\left[\frac{1}{\mu(B)} \int_{B}\left|f(x)-f_{B}\right|^{p} d \mu(x)\right]^{1 / p}\right. \\
& \left.\quad+\sum_{j=1}^{J_{0}}\left|f_{2^{j-1} B}-f_{2^{j} B}\right|+\left|f_{2^{J_{0} B}}\right|\right\}
\end{aligned}
$$




$$
\lesssim\left(1+\sum_{j=1}^{J_{0}} 2^{j \kappa \alpha}\right)\|f\|_{\mathcal{E}_{\rho}^{\alpha, p}(\mathcal{X})} \lesssim\|f\|_{\mathcal{E}_{\rho}^{\alpha, p}(\mathcal{X})}
$$

which completes the proof of Lemma 2.3.

REmARK 2.3. Let $\mathcal{X}$ be an RD-space, and let $p \in[1, \infty)$.

(i) Then Lemma 2.3 implies that $\mathcal{E}_{\rho}^{\alpha, p}(\mathcal{X})$ with $\alpha \in(-1 / p, 0)$ coincides with the so-called Morrey space (see, e.g., [24], [29] for the case $\mathcal{X}=\mathbb{R}^{d}$ ).

(ii) Let $\alpha<0$. For all $f \geq 0, f \in \mathcal{E}_{\mathcal{D}}^{\alpha, p}(\mathcal{X})$ if and only if $f \in \widetilde{\mathcal{E}}_{\mathcal{D}}^{\alpha, p}(\mathcal{X})$ and, moreover, $\|f\|_{\widetilde{\mathcal{E}}_{\mathcal{D}}^{\alpha, p}(\mathcal{X})} \sim\|f\|_{\mathcal{E}_{\mathcal{D}}^{\alpha, p}(\mathcal{X})}$. In fact, by Remark $2.2($ iii), we need to show only that for all $f \geq 0, f \in \mathcal{E}_{\mathcal{D}}^{\alpha, p}(\mathcal{X})$ implies that $f \in \widetilde{\mathcal{E}}_{\mathcal{D}}^{\alpha, p}(\mathcal{X})$ and that $\|f\|_{\widetilde{\mathcal{E}}_{\mathcal{D}}^{\alpha, p}(\mathcal{X})} \lesssim\|f\|_{\mathcal{E}_{\mathcal{D}}^{\alpha, p}(\mathcal{X})}$. By Lemma $2.3, \alpha<0$, and $f \geq 0$, we see that, for all balls $B \notin \mathcal{D}$,

$$
\int_{B}[f(x)-\underset{B}{\operatorname{essinf} f} f]^{p} d \mu(x) \leq \int_{B}[f(x)]^{p} d \mu(x) \lesssim[\mu(B)]^{1+\alpha p}\|f\|_{\mathcal{E}_{\mathcal{D}}^{\alpha, p}(\mathcal{X})}^{p},
$$

which implies the claim.

(iii) If $\mathcal{X}$ is not an RD-space, it is not clear if Lemma 2.3 still holds.

We also have the following conclusions, which are used in Sections 3 and 4.

LEMmA 2.4. Let $\alpha \in \mathbb{R}$, let $p \in[1, \infty)$, and let $\rho$ be an admissible function on $\mathcal{X}$ and $\mathcal{D}_{\rho}$ as in Remark 2.2(iv). Then there exists a positive constant $C$ such that for all $f \in \mathcal{E}_{\rho}^{\alpha, p}(\mathcal{X})$,

(i) for all balls $B \equiv B\left(x_{0}, r\right) \notin \mathcal{D}_{\rho}$,

$$
\frac{1}{\mu(B)} \int_{B}|f(z)| d \mu(z) \leq \begin{cases}C\left(\frac{\rho\left(x_{0}\right)}{r}\right)^{\alpha n}[\mu(B)]^{\alpha}\|f\|_{\mathcal{E}_{\rho}^{\alpha, p}(\mathcal{X})}, & \alpha>0 \\ C\left(1+\log \frac{\rho\left(x_{0}\right)}{r}\right)[\mu(B)]^{\alpha}\|f\|_{\mathcal{E}_{\rho}^{\alpha, p}(\mathcal{X})}, & \alpha \leq 0\end{cases}
$$

(ii) for all $x \in \mathcal{X}$ and $0<r_{1}<r_{2}$,

$$
\left|f_{B\left(x, r_{1}\right)}-f_{B\left(x, r_{2}\right)}\right| \leq \begin{cases}C\left(\frac{r_{2}}{r_{1}}\right)^{\alpha n}\left[\mu\left(B\left(x, r_{1}\right)\right)\right]^{\alpha}\|f\|_{\mathcal{E}_{\rho}^{\alpha, p}(\mathcal{X})}, & \alpha>0 \\ C\left(1+\log \frac{r_{2}}{r_{1}}\right)\left[\mu\left(B\left(x, r_{1}\right)\right)\right]^{\alpha}\|f\|_{\mathcal{E}_{\rho}^{\alpha, p}(\mathcal{X})}, & \alpha \leq 0 .\end{cases}
$$

Proof. If (ii) holds, then by the Hölder inequality, we see that for all $f \in \mathcal{E}_{\rho}^{\alpha, p}(\mathcal{X})$ and $B \notin \mathcal{D}_{\rho}$,

$$
\frac{1}{\mu(B)} \int_{B}|f(x)| d \mu(x) \leq \frac{1}{\mu(B)} \int_{B}\left|f(x)-f_{B}\right| d \mu(x)+\left|f_{B}-f_{B\left(x_{0}, \rho\left(x_{0}\right)\right)}\right|
$$




$$
\begin{aligned}
& +\frac{1}{\mu\left(B\left(x_{0}, \rho\left(x_{0}\right)\right)\right)} \int_{B\left(x_{0}, \rho\left(x_{0}\right)\right)}|f(x)| d \mu(x) \\
\lesssim & \left\{[\mu(B)]^{\alpha}+\left[\mu\left(B\left(x_{0}, \rho\left(x_{0}\right)\right)\right)\right]^{\alpha}\right\}\|f\|_{\mathcal{E}_{\rho}^{\alpha, p}(\mathcal{X})} \\
& +\left|f_{B}-f_{B\left(x_{0}, \rho\left(x_{0}\right)\right)}\right| .
\end{aligned}
$$

Then (i) follows from this fact together with $(2.1), r<\rho\left(x_{0}\right)$ (because $B \notin$ $\mathcal{D}_{\rho}$ ), and (ii).

To prove (ii), let $j_{0}$ be the smallest integer such that $2^{j_{0}} r_{1} \geq r_{2}$. Another application of (2.1) leads to

$$
\begin{aligned}
\left|f_{B\left(x, 2^{j_{0}} r_{1}\right)}-f_{B\left(x, r_{2}\right)}\right| & \lesssim \frac{1}{\mu\left(B\left(x, 2^{j}{ }^{j} r_{1}\right)\right)} \int_{B\left(x, 2^{j_{0}} r_{1}\right)}\left|f-f_{B\left(x, 2^{j_{0}} r_{1}\right)}\right| d \mu(z) \\
& \lesssim\left[\mu\left(B\left(x, 2^{j_{0}} r_{1}\right)\right)\right]^{\alpha}\|f\|_{\mathcal{E}_{\rho}^{\alpha, p}(\mathcal{X})}
\end{aligned}
$$

Similarly, we see that for all $j \in \mathbb{N} \cup\{0\}$,

$$
\left|f_{B\left(x, 2^{j} r_{1}\right)}-f_{B\left(x, 2^{j+1} r_{1}\right)}\right| \lesssim\left[\mu\left(B\left(x, 2^{j+1} r_{1}\right)\right)\right]^{\alpha}\|f\|_{\mathcal{E}_{\rho}^{\alpha, p}(\mathcal{X})}
$$

Then we have

$$
\begin{aligned}
\left|f_{B\left(x, r_{1}\right)}-f_{B\left(x, r_{2}\right)}\right| & \lesssim \sum_{j=0}^{j_{0}-1}\left|f_{B\left(x, 2^{j} r_{1}\right)}-f_{B\left(x, 2^{j+1} r_{1}\right)}\right|+\left|f_{B\left(x, 2^{j} r_{1}\right)}-f_{B\left(x, r_{2}\right)}\right| \\
& \lesssim \sum_{j=0}^{j_{0}-1}\left[\mu\left(B\left(x, 2^{j+1} r_{1}\right)\right)\right]^{\alpha}\|f\|_{\mathcal{E}_{\rho}^{\alpha, p}(\mathcal{X})} \cdot
\end{aligned}
$$

If $\alpha \in(-\infty, 0]$, from the choice of $j_{0}$, we deduce that

$$
\left|f_{B\left(x, r_{1}\right)}-f_{B\left(x, r_{2}\right)}\right| \lesssim\left(1+\log \frac{r_{2}}{r_{1}}\right)\left[\mu\left(B\left(x, r_{1}\right)\right)\right]^{\alpha}\|f\|_{\mathcal{E}_{\rho}^{\alpha, p}(\mathcal{X})}
$$

if $\alpha \in(0, \infty)$, by $(2.1)$, we obtain

$$
\left|f_{B\left(x, r_{1}\right)}-f_{B\left(x, r_{2}\right)}\right| \lesssim\left(\frac{r_{2}}{r_{1}}\right)^{\alpha n}\left[\mu\left(B\left(x, r_{1}\right)\right)\right]^{\alpha}\|f\|_{\mathcal{E}_{\rho}^{\alpha, p}(\mathcal{X})} .
$$

This finishes the proof of Lemma 2.4. 


\subsection{Localized Hardy spaces}

We begin with the notion of atoms.

Definition 2.5. Let $\mathcal{D}$ be a collection of balls in $\mathcal{X}$, let $p \in(0,1]$, and let $q \in[1, \infty] \cap(p, \infty]$.

(i) A function a supported in a ball $B \subset \mathcal{X}$ is called a $(p, q)$-atom if $\int_{\mathcal{X}} a(x) d \mu(x)=0$ and $\|a\|_{L^{q}(\mathcal{X})} \leq[\mu(B)]^{1 / q-1 / p}$ (see $\left.[4]\right)$.

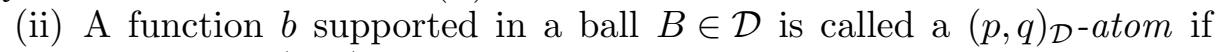
$\|b\|_{L^{q}(\mathcal{X})} \leq[\mu(B)]^{1 / q-1 / p}$.

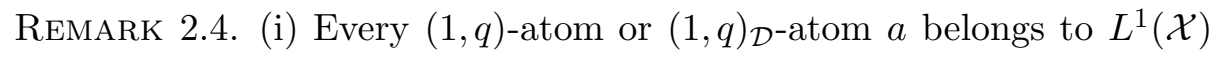
with $\|a\|_{L^{1}(\mathcal{X})} \leq 1$.

(ii) Let $p \in(0,1)$. If $a$ is a $(p, q)$-atom, then $a \in(\operatorname{Lip}(1 / p-1 ; \mathcal{X}))^{*} \subset$ $\left(\operatorname{Lip}_{\mathcal{D}}(1 / p-1 ; \mathcal{X})\right)^{*}$ and $\|a\|_{\left(\operatorname{Lip}_{\mathcal{D}}(1 / p-1 ; \mathcal{X})\right)^{*}} \leq\|a\|_{(\operatorname{Lip}(1 / p-1 ; \mathcal{X}))^{*}} \leq 1$; if $b$ is a $(p, q)_{\mathcal{D}}$-atom, then $b \in\left(\operatorname{Lip}_{\mathcal{D}}(1 / p-1 ; \mathcal{X})\right)^{*}$ and $\|b\|_{\left(\operatorname{Lip}_{\mathcal{D}}(1 / p-1 ; \mathcal{X})\right)^{*}} \leq 1$.

Definition 2.6. ([4]) Let $p \in(0,1]$, and let $q \in[1, \infty] \cap(p, \infty]$. A function $f \in L^{1}(\mathcal{X})$ or a linear functional $f \in(\operatorname{Lip}(1 / p-1 ; \mathcal{X}))^{*}$ when $p \in(0,1)$ is said to be in the Hardy space $H^{1, q}(\mathcal{X})$ when $p=1$ or $H^{p, q}(\mathcal{X})$ when $p \in(0,1)$ if there exist $(p, q)$-atoms $\left\{a_{j}\right\}_{j=1}^{\infty}$ and $\left\{\lambda_{j}\right\}_{j=1}^{\infty} \subset \mathbb{C}$ such that $f=\sum_{j \in \mathbb{N}} \lambda_{j} a_{j}$, which converges in $L^{1}(\mathcal{X})$ when $p=1$ or in $(\operatorname{Lip}(1 / p-1 ; \mathcal{X}))^{*}$ when $p \in$ $(0,1)$, and $\sum_{j \in \mathbb{N}}\left|\lambda_{j}\right|^{p}<\infty$. Moreover, the norm of $f$ in $H^{p, q}(\mathcal{X})$ with $p \in$ $(0,1]$ is defined by

$$
\|f\|_{H^{p, q}(\mathcal{X})} \equiv \inf \left\{\left(\sum_{j \in \mathbb{N}}\left|\lambda_{j}\right|^{p}\right)^{1 / p}\right\},
$$

where the infimum is taken over all the above decompositions of $f$.

Remark 2.5. Coifman and Weiss [4] proved that $H^{p, q}(\mathcal{X})$ and $H^{p, \infty}(\mathcal{X})$ coincide with equivalent norms for all $p \in(0,1]$ and $q \in[1, \infty) \cap(p, \infty)$. Thus, for all $p, q$ in this range, we denote $H^{p, q}(\mathcal{X})$ simply by $H^{p}(\mathcal{X})$. We remark that Coifman and Weiss [4] also proved that the dual space of $H^{p}(\mathcal{X})$ is $\operatorname{BMO}(\mathcal{X})$ when $p=1$ or $\operatorname{Lip}(1 / p-1 ; \mathcal{X})$ when $p \in(0,1)$.

Definition 2.7. Let $\mathcal{D}$ be a collection of balls in $\mathcal{X}$, let $p \in(0,1]$, and let $q \in[1, \infty] \cap(p, \infty]$. A function $f \in L^{1}(\mathcal{X})$ or a linear functional $f \in$ $\left(\operatorname{Lip}_{\mathcal{D}}(1 / p-1 ; \mathcal{X})\right)^{*}$ when $p \in(0,1)$ is said to be in $H_{\mathcal{D}}^{1, q}(\mathcal{X})$ when $p=1$ or $H_{\mathcal{D}}^{p, q}(\mathcal{X})$ when $p \in(0,1)$ if there exist $\left\{\lambda_{j}\right\}_{j \in \mathbb{N}},\left\{\nu_{k}\right\}_{k \in \mathbb{N}} \subset \mathbb{C},(p, q)$-atoms $\left\{a_{j}\right\}_{j \in \mathbb{N}}$, and $(p, q)_{\mathcal{D}}$-atoms $\left\{b_{k}\right\}_{k \in \mathbb{N}}$ such that

$$
f=\sum_{j \in \mathbb{N}} \lambda_{j} a_{j}+\sum_{k \in \mathbb{N}} \nu_{k} b_{k},
$$


which converges in $L^{1}(\mathcal{X})$ when $p=1$ or in $\left(\operatorname{Lip}_{\mathcal{D}}(1 / p-1 ; \mathcal{X})\right)^{*}$ when $p \in$ $(0,1]$, and $\sum_{j \in \mathbb{N}}\left|\lambda_{j}\right|^{p}+\sum_{k=1}^{\infty}\left|\nu_{k}\right|^{p}<\infty$. Moreover, the norm of $f$ in $H_{\mathcal{D}}^{p, q}(\mathcal{X})$ is defined by

$$
\|f\|_{H_{\mathcal{D}}^{p, q}(\mathcal{X})} \equiv \inf \left\{\left(\sum_{j \in \mathbb{N}}\left|\lambda_{j}\right|^{p}+\sum_{k \in \mathbb{N}}\left|\nu_{k}\right|^{p}\right)^{1 / p}\right\},
$$

where the infimum is taken over all the above decompositions of $f$.

Remark 2.6. Let $p \in(0,1]$, and let $q \in[1, \infty] \cap(p, \infty]$. It is easy to see that $H^{p, q}(\mathcal{X}) \subset H_{\mathcal{D}}^{p, q}(\mathcal{X})$.

Using Remark 2.6, we have the following conclusion.

Lemma 2.5. Let $\mathcal{D}$ be a collection of balls in $\mathcal{X}$, let $p \in(0,1]$, and let $q \in[1, \infty) \cap(p, \infty)$. Then $H_{\mathcal{D}}^{p, q}(\mathcal{X})=H_{\mathcal{D}}^{p, \infty}(\mathcal{X})$ with equivalent norms.

Proof. Notice that $(p, \infty)$-atoms and $(p, \infty)_{\mathcal{D}}$-atoms are $(p, q)$-atoms and $(p, q)_{\mathcal{D}}$-atoms, respectively. Then from Definition 2.7 , it follows that $H_{\mathcal{D}}^{p, \infty}(\mathcal{X}) \subset H_{\mathcal{D}}^{p, q}(\mathcal{X})$

Conversely, let $f \in H_{\mathcal{D}}^{p, q}(\mathcal{X})$. Then by Definition 2.7, there exist $\left\{\lambda_{j}\right\}_{j \in \mathbb{N}}$, $\left\{\nu_{k}\right\}_{k \in \mathbb{N}} \subset \mathbb{C},(p, q)$-atoms $\left\{a_{j}\right\}_{j \in \mathbb{N}}$, and $(p, q)_{\mathcal{D}}$-atoms $\left\{b_{k}\right\}_{k \in \mathbb{N}}$ such that

$$
f=\sum_{j \in \mathbb{N}} \lambda_{j} a_{j}+\sum_{k \in \mathbb{N}} \nu_{k} b_{k}
$$

which converges in $L^{1}(\mathcal{X})$ when $p=1$ or in $\left(\operatorname{Lip}_{\mathcal{D}}(1 / p-1 ; \mathcal{X})\right)^{*}$ when $p \in$ $(0,1]$, and

$$
\sum_{j \in \mathbb{N}}\left|\lambda_{j}\right|^{p}+\sum_{k \in \mathbb{N}}\left|\nu_{k}\right|^{p} \lesssim\|f\|_{H_{\mathcal{D}}^{p, q}(\mathcal{X})}^{p}
$$

For $k \in \mathbb{N}$, assume that $\operatorname{supp} b_{k} \subset B_{k} \in \mathcal{D}$, and let $c_{k} \equiv\left[b_{k}-\left(b_{k}\right)_{B_{k}} \chi_{B_{k}}\right] / 2$. Then it follows from Definition 2.5 that there exists a positive constant $\widetilde{C}$ such that $\left\{\widetilde{C} c_{k}\right\}_{k \in \mathbb{N}}$ are $(p, q)$-atoms, $\left\{\left(b_{k}\right)_{B_{k}} \chi_{B_{k}}\right\}_{k \in \mathbb{N}}$ are $(p, \infty)_{\mathcal{D}}$-atoms, and $b_{k}=2 c_{k}+\left(b_{k}\right)_{B_{k}} \chi_{B_{k}}$. Moreover,

$$
f=\sum_{j \in \mathbb{N}} \lambda_{j} a_{j}+\sum_{k \in \mathbb{N}} 2 \nu_{k} c_{k}+\sum_{k \in \mathbb{N}} \nu_{k}\left(b_{k}\right)_{B_{k}} \chi_{B_{k}},
$$

which converges in $L^{1}(\mathcal{X})$ when $p=1$ or in $\left(\operatorname{Lip}_{\mathcal{D}}(1 / p-1 ; \mathcal{X})\right)^{*}$ when $p \in$ $(0,1)$. By Remark 2.4(ii) and (2.6), we see that $\sum_{j \in \mathbb{N}} \lambda_{j} a_{j}+2 \sum_{k \in \mathbb{N}} \nu_{k} c_{k}$ also 
converges in $L^{1}(\mathcal{X})$ when $p=1$ or in $(\operatorname{Lip}(1 / p-1 ; \mathcal{X}))^{*}$ when $p \in(0,1)$. Let $g \equiv \sum_{j \in \mathbb{N}} \lambda_{j} a_{j}+2 \sum_{k \in \mathbb{N}} \nu_{k} c_{k}$. Then Definition 2.6 together with Remark 2.5 implies that $g \in H^{p, q}(\mathcal{X})=H^{p, \infty}(\mathcal{X})$. From this, Remark 2.6, and (2.6), we deduce that $g \in H^{p, \infty}(\mathcal{X}) \subset H_{\mathcal{D}}^{p, \infty}(\mathcal{X})$ and that

$$
\|g\|_{H_{\mathcal{D}}^{p, \infty}(\mathcal{X})} \lesssim\|g\|_{H^{p, \infty}(\mathcal{X})} \lesssim\|g\|_{H^{p, q}(\mathcal{X})} \lesssim\|f\|_{H_{\mathcal{D}}^{p, q}(\mathcal{X})}
$$

which further implies that $f \in H_{\mathcal{D}}^{p, \infty}(\mathcal{X})$ and, by $(2.6)$, that

$$
\begin{aligned}
\|f\|_{H_{\mathcal{D}}^{p, \infty}(\mathcal{X})} & \lesssim\|g\|_{H_{\mathcal{D}}^{p, \infty}(\mathcal{X})}+\left\|\sum_{k \in \mathbb{N}} \nu_{k}\left(b_{k}\right)_{B_{k}} \chi_{B_{k}}\right\|_{H_{\mathcal{D}}^{p, \infty}(\mathcal{X})} \\
& \lesssim\|f\|_{H_{\mathcal{D}}^{p, q}(\mathcal{X})}+\left\{\sum_{k \in \mathbb{N}}\left|\nu_{k}\right|^{p}\right\}^{1 / p} \lesssim\|f\|_{H_{\mathcal{D}}^{p, q}(\mathcal{X})}
\end{aligned}
$$

This finishes the proof of Lemma 2.5.

REMARK 2.7. (i) Let $\mathcal{D}$ be a collection of balls in $\mathcal{X}$, let $p \in(0,1]$, and let $q \in[1, \infty] \cap(p, \infty]$. In what follows, based on Lemma 2.5 , we denote $H_{\mathcal{D}}^{p, q}(\mathcal{X})$ simply by $H_{\mathcal{D}}^{p}(\mathcal{X})$.

(ii) Let $L_{b}^{\infty}(\mathcal{X})$ be the set of all functions of $L^{\infty}(\mathcal{X})$ with bounded support. Then from Definitions 2.6 and 2.7, it follows that $L_{b}^{\infty}(\mathcal{X}) \cap H_{\mathcal{D}}^{p}(\mathcal{X})$ is dense in $H_{\mathcal{D}}^{p}(\mathcal{X})$ and that $L_{b}^{\infty}(\mathcal{X}) \cap H^{p}(\mathcal{X})$ is dense in $H^{p}(\mathcal{X})$.

TheOREM 2.1. Let $\mathcal{D}$ be a collection of balls in $\mathcal{X}$, and let $p \in(0,1]$. Then $\mathcal{E}_{\mathcal{D}}^{1 / p-1,1}(\mathcal{X})=\left(H_{\mathcal{D}}^{p}(\mathcal{X})\right)^{*}$

Proof. We first prove $\mathcal{E}_{\mathcal{D}}^{1 / p-1,1}(\mathcal{X}) \subset\left(H_{\mathcal{D}}^{p, \infty}(\mathcal{X})\right)^{*}$ for $p \in(0,1]$. Let $f \in$ $\mathcal{E}_{\mathcal{D}}^{1 / p-1,1}(\mathcal{X})$. For all $(p, \infty)$-atoms a supported in $B \notin \mathcal{D}$, by Definition $2.5(\mathrm{i})$ we have

$$
\begin{aligned}
\left|\int_{\mathcal{X}} f(x) a(x) d \mu(x)\right| & =\left|\int_{\mathcal{X}}\left[f(x)-f_{B}\right] a(x) d \mu(x)\right| \\
& \leq \frac{1}{[\mu(B)]^{1 / p}} \int_{B}\left|f(x)-f_{B}\right| d \mu(x) \leq\|f\|_{\mathcal{E}_{\mathcal{D}}^{1 / p-1,1}(\mathcal{X})} .
\end{aligned}
$$

For all $(p, \infty)_{\mathcal{D}}$-atoms $b$ supported in $B \in \mathcal{D}$, we also obtain

$$
\left|\int_{\mathcal{X}} f(x) b(x) d \mu(x)\right| \leq \frac{1}{[\mu(B)]^{1 / p}} \int_{B}|f(x)| d \mu(x) \leq\|f\|_{\mathcal{E}_{\mathcal{D}}^{1 / p-1,1}(\mathcal{X})} .
$$


Let $N \in \mathbb{N}$, and let $f_{N} \equiv \max \{\min \{f, N\},-N\}$. We claim that $f_{N} \in$ $\mathcal{E}_{\mathcal{D}}^{1 / p-1,1}(\mathcal{X})$ and that

$$
\left\|f_{N}\right\|_{\mathcal{E}_{\mathcal{D}}^{1 / p-1,1}(\mathcal{X})} \leq \frac{9}{4}\|f\|_{\mathcal{E}_{\mathcal{D}}^{1 / p-1,1}(\mathcal{X})} .
$$

In fact, if $B \in \mathcal{D}$, then

$$
\frac{1}{[\mu(B)]^{1 / p}} \int_{B}\left|f_{N}(x)\right| d \mu(x) \leq \frac{1}{[\mu(B)]^{1 / p}} \int_{B}|f(x)| d \mu(x) \leq\|f\|_{\mathcal{E}_{\mathcal{D}}^{1 / p-1,1}(\mathcal{X})} .
$$

Let $B \notin \mathcal{D}$. For all $f, h \in \mathcal{E}_{\mathcal{D}}^{1 / p-1,1}(\mathcal{X})$ and $g \equiv \max \{f, h\}$, we have $g=$ $(f+h+|f-h|) / 2$ and

$$
\begin{aligned}
& \frac{1}{[\mu(B)]^{1 / p}} \int_{B}\left|g(x)-g_{B}\right| d \mu(x) \\
& \leq \frac{1}{2[\mu(B)]^{1 / p}} \int_{B}\left|f(x)-f_{B}\right| d \mu(x)+\frac{1}{2[\mu(B)]^{1 / p}} \int_{B}\left|h(x)-h_{B}\right| d \mu(x) \\
& \quad+\frac{1}{[\mu(B)]^{1 / p}} \int_{B}\left|(f-h)(x)-(f-h)_{B}\right| d \mu(x) \\
& \leq \leq \frac{3}{2}\left(\|f\|_{\mathcal{E}^{1 / p-1,1}(\mathcal{X})}+\|h\|_{\mathcal{E}^{1 / p-1,1}(\mathcal{X})}\right) .
\end{aligned}
$$

Similarly, for all $B \notin \mathcal{D}, f, h \in \mathcal{E}_{\mathcal{D}}^{1 / p-1,1}(\mathcal{X})$, and $\widetilde{g} \equiv \min \{f, h\}$, we have

$$
\frac{1}{[\mu(B)]^{1 / p}} \int_{B}\left|\widetilde{g}(x)-\widetilde{g}_{B}\right| d \mu(x) \leq \frac{3}{2}\left(\|f\|_{\mathcal{E}^{1 / p-1,1}(\mathcal{X})}+\|h\|_{\mathcal{E}^{1 / p-1,1}(\mathcal{X})}\right) .
$$

If $h \equiv N$ or $h \equiv-N$, then $\|h\|_{\mathcal{E}^{1 / p-1,1}(\mathcal{X})}=0$. By these facts and the definition of $f_{N}$, we have that for all $B \notin \mathcal{D}$,

$$
\frac{1}{[\mu(B)]^{1 / p}} \int_{B}\left|f_{N}(x)-\left(f_{N}\right)_{B}\right| d \mu(x) \leq \frac{9}{4}\|f\|_{\mathcal{E}_{\mathcal{D}}^{1 / p-1,1}(\mathcal{X})},
$$

which implies the claim.

For all $g \in L_{b}^{\infty}(\mathcal{X}) \cap H_{\mathcal{D}}^{p, \infty}(\mathcal{X})$, since $f g \in L^{1}(\mathcal{X})$, we define $\ell(g) \equiv$ $\int_{\mathcal{X}} f(x) g(x) d \mu(x)$ and $\ell_{N}(g) \equiv \int_{\mathcal{X}} f_{N}(x) g(x) d \mu(x)$. Moreover, there exist $\left\{\lambda_{j}\right\},\left\{\nu_{k}\right\}_{k \in \mathbb{N}} \subset \mathbb{C},(p, \infty)$-atoms $\left\{a_{j}\right\}_{j \in \mathbb{N}}$, and $(p, \infty)_{\mathcal{D}}$-atoms $\left\{b_{k}\right\}_{k \in \mathbb{N}}$ such that

$$
g=\sum_{j \in \mathbb{N}} \lambda_{j} a_{j}+\sum_{k \in \mathbb{N}} \nu_{k} b_{k}
$$


which converges in $L^{1}(\mathcal{X})$ when $p=1$ or in $\left(\operatorname{Lip}_{\mathcal{D}}(1 / p-1 ; \mathcal{X})\right)^{*}$ when $p \in$ $(0,1)$, and

$$
\sum_{j \in \mathbb{N}}\left|\lambda_{j}\right|^{p}+\sum_{k \in \mathbb{N}}\left|\nu_{k}\right|^{p} \leq 2\|g\|_{H_{\mathcal{D}}^{p, \infty}(\mathcal{X})}^{p}
$$

By $f_{N} \in \mathcal{E}_{\mathcal{D}}^{1 / p-1,1}(\mathcal{X})$ and $g \in H_{\mathcal{D}}^{p, \infty}(\mathcal{X})$, we have

$$
\ell_{N}(g)=\sum_{j \in \mathbb{N}} \int_{\mathcal{X}} f_{N}(x) \lambda_{j} a_{j}(x) d \mu(x)+\sum_{k \in \mathbb{N}} \int_{\mathcal{X}} f_{N}(x) \nu_{k} b_{k}(x) d \mu(x),
$$

from which, together with (2.7), (2.8), and Remark 2.4(ii), it follows that

$$
\left|\ell_{N}(g)\right| \lesssim\left\|f_{N}\right\|_{\mathcal{E}_{\mathcal{D}}^{1 / p-1,1}(\mathcal{X})}\left\{\sum_{j \in \mathbb{N}}\left|\lambda_{j}\right|+\sum_{k \in \mathbb{N}}\left|\nu_{k}\right|\right\} \lesssim\|f\|_{\mathcal{E}_{\mathcal{D}}^{1 / p-1,1}(\mathcal{X})}\|g\|_{H_{\mathcal{D}}^{p, \infty}(\mathcal{X})} .
$$

By this and the Lebesgue dominated theorem, we have

$$
|\ell(g)|=\lim _{N \rightarrow \infty}\left|\int_{\mathcal{X}} f_{N}(x) g(x) d \mu(x)\right| \lesssim\|f\|_{\mathcal{E}_{\mathcal{D}}^{1 / p-1,1}(\mathcal{X})}\|g\|_{H_{\mathcal{D}}^{p, \infty}(\mathcal{X})},
$$

which together with the density of $L_{b}^{\infty}(\mathcal{X}) \cap H_{\mathcal{D}}^{p, \infty}(\mathcal{X})$ in $H_{\mathcal{D}}^{p, \infty}(\mathcal{X})$ (see Remark 2.7(ii)) implies that $\ell \in\left(H_{\mathcal{D}}^{p, \infty}(\mathcal{X})\right)^{*}$ and that $\|\ell\|_{\left(H_{\mathcal{D}}^{p, \infty}(\mathcal{X})\right)^{*}} \lesssim$ $\|f\|_{\mathcal{E}_{\mathcal{D}}^{1 / p-1,1}(\mathcal{X})}$. Thus,

$$
\mathcal{E}_{\mathcal{D}}^{1 / p-1,1}(\mathcal{X}) \subset\left(H_{\mathcal{D}}^{p}(\mathcal{X})\right)^{*}
$$

We now prove that $\left(H_{\mathcal{D}}^{p, 2}(\mathcal{X})\right)^{*} \subset \mathcal{E}_{\mathcal{D}}^{1 / p-1,2}(\mathcal{X})$. Let $\ell \in\left(H_{\mathcal{D}}^{p, 2}(\mathcal{X})\right)^{*}$. Since $H^{p, 2}(\mathcal{X}) \subset H_{\mathcal{D}}^{p, 2}(\mathcal{X})$, then $\ell \in\left(H^{p, 2}(\mathcal{X})\right)^{*}=\mathcal{E}^{1 / p-1,2}(\mathcal{X})$ (see Remarks 2.5, $2.1(\mathrm{i})$, and $2.1(\mathrm{iii}))$. Hence there exists $\tilde{f} \in \mathcal{E}^{1 / p-1,2}(\mathcal{X})$ such that, for all constants $C$ and $g \in L^{2}(\mathcal{X})$ satisfying that $\int_{\mathcal{X}} g(x) d \mu(x)=0$ and $\operatorname{supp}(g)$ is bounded,

$$
\ell(g)=\int_{\mathcal{X}} \tilde{f}(x) g(x) d \mu(x)=\int_{\mathcal{X}}(\widetilde{f}(x)+C) g(x) d \mu(x)
$$

and $\|\widetilde{f}\|_{\mathcal{E}^{1 / p-1,2}(\mathcal{X})} \lesssim\|\ell\|_{\left(H^{p, 2}(\mathcal{X})\right)^{*}} \lesssim\|\ell\|_{\left(H_{\mathcal{D}}^{p, 2}(\mathcal{X})\right)^{*}}$. We then need to choose a suitable constant $C$ such that $f \equiv \widetilde{f}+C \in \mathcal{E}_{\mathcal{D}}^{1 / p-1,2}(\mathcal{X})$. 
Observe that for all constants $\widetilde{C}, \widetilde{f}+\widetilde{C} \in \mathcal{E}^{1 / p-1,2}(\mathcal{X})$. Then by Lemma $2.1(\mathrm{i})$, to show $f \in \mathcal{E}_{\mathcal{D}}^{1 / p-1,2}(\mathcal{X})$ and $\|f\|_{\mathcal{E}_{\mathcal{D}}^{1 / p-1,2}(\mathcal{X})} \lesssim\|\ell\|_{\left(H_{\mathcal{D}}^{p, 2}(\mathcal{X})\right)^{*}}$, it suffices to show that for all $B \in \mathcal{D}$,

$$
\left|f_{B}\right|[\mu(B)]^{1-1 / p} \lesssim\|\ell\|_{\left(H_{\mathcal{D}}^{p, 2}(\mathcal{X})\right)^{*}}
$$

To this end, for any $B \in \mathcal{D}$, let $L^{2}(B) \equiv\left\{f \in L^{2}(\mathcal{X}): \operatorname{supp}(f) \subset B\right\}$, and let $L_{0}^{2}(B) \equiv\left\{f \in L^{2}(B): \int_{\mathcal{X}} f(x) d \mu(x)=0\right\}$. Then for any $g \in L^{2}(B)$, the function $g[\mu(B)]^{1 / 2-1 / p}\|g\|_{L^{2}(B)}^{-1}$ is a $(p, 2)_{\mathcal{D}^{-}}$-atom supported in $B$, and

$$
|\ell(g)| \leq\|\ell\|_{\left(H_{\mathcal{D}}^{p, 2}(\mathcal{X})\right)^{*}}\|g\|_{H_{\mathcal{D}}^{p, 2}(\mathcal{X})} \leq[\mu(B)]^{1 / p-1 / 2}\|\ell\|_{\left(H_{\mathcal{D}}^{p, 2}(\mathcal{X})\right)^{*}}\|g\|_{L^{2}(B)},
$$

which implies that $\ell \in\left(L^{2}(B)\right)^{*}=L^{2}(B)$. By this together with the Riesz representation theorem, there exists a function $f^{B} \in L^{2}(B)$ such that for all $g \in L^{2}(B), \ell(g)=\int_{B} f^{B}(x) g(x) d \mu(x)$ and

$$
\left\|f^{B}\right\|_{L^{2}(B)} \leq[\mu(B)]^{1 / p-1 / 2}\|\ell\|_{\left(H_{\mathcal{D}}^{p, 2}(\mathcal{X})\right)^{*}}
$$

Moreover, from this fact and (2.10), we deduce that for all $g \in L_{0}^{2}(B)$, $\int_{\mathcal{X}}\left[f^{B}(x)-\widetilde{f}(x)\right] g(x) d \mu(x)=0$, which further implies that $f^{B}-\widetilde{f}=0$ in $\left[L_{0}^{2}(B)\right]^{*}$. Recall that $\left[L_{0}^{2}(B)\right]^{*}=L^{2}(B) / \mathbb{C}$ (the space of functions $f \in L^{2}(B)$ modulo constant functions) and $f=0$ in $L^{2}(B) / \mathbb{C}$ if and only if $f$ is a constant (see [4, page 633]). Using these facts, we have that $f^{B}-\widetilde{f}$ is a constant $C_{B}$.

Now it suffices to verify that, for all balls $B, S \in \mathcal{D}$, we have $C_{B}=C_{S}$. Observe that $g \equiv\left\{[\mu((1 / 2) B)]^{-1} \chi_{(1 / 2) B}-[\mu((1 / 2) S)]^{-1} \chi_{(1 / 2) S}\right\}$ is a multiple of certain $(p, 2)$-atom and that $[\mu((1 / 2) B)]^{-1} \chi_{(1 / 2) B}$ and $[\mu((1 / 2) S)]^{-1} \chi_{(1 / 2) S}$ are multiplies of $(p, 2)_{\mathcal{D}}$-atoms. Therefore, from the fact that $f^{B}-C_{B}=\widetilde{f}=$ $f^{S}-C_{S}$ and (2.10), it follows that

$$
\begin{aligned}
\ell(g) & =\ell\left(\left[\mu\left(\frac{1}{2} B\right)\right]^{-1} \chi_{(1 / 2) B}\right)-\ell\left(\left[\mu\left(\frac{1}{2} S\right)\right]^{-1} \chi_{(1 / 2) S}\right) \\
& =\frac{1}{\mu\left(\frac{1}{2} B\right)} \int_{B} f^{B}(x) \chi_{(1 / 2) B}(x) d \mu(x)-\frac{1}{\mu\left(\frac{1}{2} S\right)} \int_{S} f^{S}(x) \chi_{(1 / 2) S}(x) d \mu(x) \\
& =\int_{B \cup S} \tilde{f}(x) g(x) d \mu(x)+C_{B}-C_{S}=\ell(g)+C_{B}-C_{S},
\end{aligned}
$$

which implies that $C_{B}=C_{S}$. Denote the constant as above by $\widetilde{C}$, and define $f \equiv \widetilde{f}+\widetilde{C}$. Then by this, (2.12), and the Hölder inequality, we have that for 
all $B \in \mathcal{D}$,

$$
\left|f_{B}\right|[\mu(B)]^{1-1 / p}=\left|\left(f^{B}\right)_{B}\right|[\mu(B)]^{1-1 / p} \lesssim\|\ell\|_{\left(H_{\mathcal{D}}^{p, 2}(\mathcal{X})\right)^{*}}
$$

This implies (2.11), from which and from Lemma 2.1(i) we further deduce that $f \in \mathcal{E}_{\mathcal{D}}^{1 / p-1,2}(\mathcal{X})$ and that $\|f\|_{\mathcal{E}_{\mathcal{D}}^{1 / p-1,2}(\mathcal{X})} \lesssim\|\ell\|_{\left(H_{\mathcal{D}}^{p, 2}(\mathcal{X})\right)^{*}}$. Thus, $\left(H_{\mathcal{D}}^{p}(\mathcal{X})\right)^{*} \subset$ $\mathcal{E}_{\mathcal{D}}^{1 / p-1,2}(\mathcal{X})$, which together with Lemma 2.2 and $(2.9)$ then completes the proof of Theorem 2.1.

\section{$\S 3$. Boundedness of the radial and the Poisson maximal functions}

This section is devoted to the boundedness of the radial and the Poisson maximal functions from $\mathcal{E}_{\rho}^{\alpha, p}(\mathcal{X})$ to $\widetilde{\mathcal{E}}_{\rho}^{\alpha, p}(\mathcal{X})$. We start with the notion of the radial maximal function.

Definition 3.1. Let $\rho$ be an admissible function on $\mathcal{X}$, and let $\left\{T_{t}\right\}_{t>0}$ be a family of linear integral operators on $L^{2}(\mathcal{X})$. Moreover, assume that there exist positive constants $C, \gamma, \delta_{1}, \delta_{2}$, and $\beta$ satisfying that for all $t \in(0, \infty)$ and $x, x^{\prime}, y \in \mathcal{X}$ with $d\left(x, x^{\prime}\right) \leq t / 2$,

$$
\begin{gathered}
\left|T_{t}(x, y)\right| \leq C \frac{1}{V_{t}(x)+V(x, y)}\left(\frac{t}{t+d(x, y)}\right)^{\gamma}\left(\frac{\rho(x)}{t+\rho(x)}\right)^{\delta_{1}} \\
\left|T_{t}(x, y)-T_{t}\left(x^{\prime}, y\right)\right| \leq C \frac{1}{V_{t}(x)+V(x, y)}\left(\frac{t}{t+d(x, y)}\right)^{\gamma}\left(\frac{d\left(x, x^{\prime}\right)}{t}\right)^{\beta} \\
\left|1-T_{t}(1)(x)\right| \leq C\left(\frac{t}{t+\rho(x)}\right)^{\delta_{2}}
\end{gathered}
$$

Let $\left\{T_{t}\right\}_{t>0}$ be as in Definition 3.1. For all $f \in L_{\text {loc }}^{1}(\mathcal{X})$, the radial maximal function $T^{+}$is defined by

$$
T^{+}(f) \equiv \sup _{t>0}\left|T_{t}(f)\right|
$$

Then we have the following result.

TheOREM 3.1. Let $\alpha \in(-\infty, \gamma / n) \cap\left(-\infty, \min \left\{\beta /(2 n), \delta_{1} / n, \delta_{2} /(2 n)\right\}\right]$, let $p \in(1, \infty)$, and let $\rho$ be an admissible function. If $\left\{T_{t}\right\}_{t>0}$ satisfies (3.1)(3.3), then there exists a positive constant $C$ such that for all $f \in \mathcal{E}_{\rho}^{\alpha, p}(\mathcal{X})$, $T^{+}(f) \in \widetilde{\mathcal{E}}_{\rho}^{\alpha, p}(\mathcal{X})$ and

$$
\left\|T^{+}(f)\right\|_{\widetilde{\mathcal{E}}_{\rho}^{\alpha, p}(\mathcal{X})} \leq C\|f\|_{\mathcal{E}_{\rho}^{\alpha, p}(\mathcal{X})} .
$$


Proof. We consider only the case that $\alpha \in(0, \gamma / n) \cap\left(0, \min \left\{\beta /(2 n), \delta_{1} / n\right.\right.$, $\left.\left.\delta_{2} /(2 n)\right\}\right]$; the proof for $\alpha \in(-\infty, 0]$ is similar but easier. By the homogeneity of $\|\cdot\|_{\mathcal{E}_{\rho}^{\alpha, p}(\mathcal{X})}$ and $\|\cdot\|_{\widetilde{\mathcal{E}}_{\rho}^{\alpha, p}(\mathcal{X})}$, we assume that $f \in \mathcal{E}_{\rho}^{\alpha, p}(\mathcal{X})$ and $\|f\|_{\mathcal{E}_{\rho}^{\alpha, p}(\mathcal{X})}=1$.

Let $\mathcal{D}_{\rho}$ be as in Remark $2.2(\mathrm{iv})$, and let $B \equiv B\left(x_{0}, r\right) \in \mathcal{D}_{\rho}$. Observe that $T^{+}(f) \lesssim \mathrm{HL}(f)$, where for all $x \in \mathcal{X}$ and $f \in L_{\text {loc }}^{1}(\mathcal{X}), \operatorname{HL}(f)$ denotes the Hardy-Littlewood maximal function of $f$ defined by

$$
\operatorname{HL}(f)(x) \equiv \sup _{B \ni x} \frac{1}{\mu(B)} \int_{B}|f(y)| d \mu(y) .
$$

Recall that HL is bounded on $L^{p}(\mathcal{X})$ for $p \in(1, \infty]$. Therefore $T^{+}$is bounded on $L^{p}(\mathcal{X})$ for all $p \in(1, \infty]$. By this fact together with $(2.1)$, we see that

$$
\int_{B}\left[T^{+}\left(f \chi_{2 B}\right)(x)\right]^{p} d \mu(x) \lesssim \int_{2 B}|f(x)|^{p} d \mu(x) \lesssim[\mu(B)]^{1+\alpha p} .
$$

If $t \in(0, r)$, then by $(3.1),(2.1)$, the Hölder inequality, and $\gamma>\alpha n$, we have

$$
\begin{aligned}
& \left|T_{t}\left(f \chi_{(2 B)^{\mathrm{C}}}\right)(x)\right| \\
& \quad \lesssim \int_{(2 B)^{\complement}} \frac{1}{V_{t}(x)+V(x, y)}\left(\frac{t}{t+d(x, y)}\right)^{\gamma}|f(y)| d \mu(y) \\
& \quad \lesssim \sum_{j=1}^{\infty} 2^{-j \gamma}\left(\frac{1}{\mu\left(2^{j+1} B\right)} \int_{2^{j+1} B}|f(y)|^{p} d \mu(y)\right)^{1 / p} \\
& \quad \lesssim \sum_{j=1}^{\infty} 2^{-j \gamma}\left[\mu\left(2^{j+1} B\right)\right]^{\alpha} \lesssim[\mu(B)]^{\alpha} \sum_{j=1}^{\infty} 2^{-j(\gamma-\alpha n)} \\
& \quad \lesssim[\mu(B)]^{\alpha} .
\end{aligned}
$$

Let $t \in[r, \infty)$. By $(2.2)$, we see that for all $a \in(0, \infty)$, there exists a constant $\widetilde{C}_{a} \in[1, \infty)$ such that for all $x, y \in \mathcal{X}$ with $d(x, y) \leq a \rho(x)$,

$$
\rho(y) / \widetilde{C}_{a} \leq \rho(x) \leq \widetilde{C}_{a} \rho(y)
$$

Recall that $B \in \mathcal{D}_{\rho}$, which is equivalent to that $r \geq \rho\left(x_{0}\right)$. These facts imply that for all $x \in B, \rho(x) \lesssim r$. By this together with (3.1), (2.1), the Hölder 
inequality, and the facts that $\gamma>\alpha n$ and $\delta_{1} \geq \alpha n$, we have that for all $t \in[r, \infty)$ and $x \in B$,

$$
\begin{aligned}
\mid T_{t} & \left(f \chi_{(2 B)^{\mathrm{C}}}\right)(x) \mid \\
& \lesssim \int_{(2 B)^{\mathrm{C}}} \frac{|f(y)|}{V_{t}(x)+V(x, y)}\left(\frac{t}{t+d(x, y)}\right)^{\gamma}\left(\frac{\rho(x)}{t+\rho(x)}\right)^{\delta_{1}} d \mu(y) \\
& \lesssim\left(\frac{\rho(x)}{t+\rho(x)}\right)^{\delta_{1}} \sum_{j=1}^{\infty} 2^{-j \gamma} \frac{1}{V_{2^{j-1} t}(x)} \int_{d(x, y)<2^{j} t}|f(y)| d \mu(y) \\
& \lesssim\left(\frac{\rho(x)}{t+\rho(x)}\right)^{\delta_{1}} \sum_{j=1}^{\infty} 2^{-j \gamma}\left(\frac{1}{V_{2^{j+1}}\left(x_{0}\right)} \int_{d\left(x_{0}, y\right)<2^{j+1} t}|f(y)|^{p} d \mu(y)\right)^{1 / p} \\
& \lesssim\left(\frac{\rho(x)}{t+\rho(x)}\right)^{\delta_{1}} \sum_{j=1}^{\infty} 2^{-j \gamma}\left[V_{2^{j+1} t}\left(x_{0}\right)\right]^{\alpha} \\
& \lesssim\left(\frac{\rho(x)}{t+\rho(x)}\right)^{\delta_{1}}\left(\frac{t}{r}\right)^{\alpha n}[\mu(B)]^{\alpha} \sum_{j=1}^{\infty} 2^{-j(\gamma-\alpha n)} \\
& \lesssim[\mu(B)]^{\alpha} \cdot
\end{aligned}
$$

Combining this and (3.5) yields that for all $t \in(0, \infty)$,

$$
\int_{B}\left[T^{+}\left(f \chi_{(2 B)^{\complement}}\right)(x)\right]^{p} d \mu(x) \lesssim[\mu(B)]^{1+\alpha p},
$$

which together with (3.4) gives us that

$$
\int_{B}\left[T^{+}(f)(x)\right]^{p} d \mu(x) \lesssim[\mu(B)]^{1+\alpha p}
$$

This also implies that $T^{+}(f)(x)<\infty$ for $\mu$ almost every $x \in \mathcal{X}$.

It remains to show that for all $B \equiv B\left(x_{0}, r\right) \notin \mathcal{D}_{\rho}$,

$$
\int_{B}\left[T^{+}(f)(x)-\underset{B}{\operatorname{essinf}} T^{+}(f)\right]^{p} d \mu(x) \lesssim[\mu(B)]^{1+\alpha p} .
$$

Let $f_{1} \equiv\left(f-f_{B}\right) \chi_{2 B}$, let $f_{2} \equiv\left(f-f_{B}\right) \chi_{(2 B)^{\complement}}$, let $B_{1} \equiv\left\{x \in B: T_{r}^{+}(f)(x) \geq\right.$ $\left.T_{\infty}^{+}(f)(x)\right\}$, and let $B_{2} \equiv B \backslash B_{1}$, where $T_{r}^{+}(f) \equiv \sup _{0<t<4 r}\left|T_{t}(f)\right|$ and 
$T_{\infty}^{+}(f) \equiv \sup _{t \geq 4 r}\left|T_{t}(f)\right|$. We have

$$
\begin{aligned}
\int_{B} & {\left[T^{+}(f)(x)-\underset{B}{\operatorname{essinf}} T^{+}(f)\right]^{p} d \mu(x) } \\
\lesssim & \int_{B_{1}}\left[T_{r}^{+}(f)(x)-\underset{B}{\operatorname{essinf}}\left|T_{r}(f)\right|\right]^{p} d \mu(x) \\
& \quad+\int_{B_{2}}\left[T_{\infty}^{+}(f)(x)-\underset{B}{\operatorname{essinf}} T_{\infty}^{+}(f)\right]^{p} d \mu(x) \\
\lesssim & \int_{B}\left[T_{r}^{+}\left(f_{1}\right)(x)\right]^{p} d \mu(x)+\mu(B) \sup _{x, y \in B} \sup _{0<t<4 r}\left|T_{t}\left(f_{B}\right)(x)-T_{r}(f)(y)\right|^{p} \\
& \quad+\int_{B}\left[T_{r}^{+}\left(f_{2}\right)(x)\right]^{p} d \mu(x)+\mu(B) \sup _{x, y \in B} \sup _{t \geq 4 r}\left|T_{t}(f)(x)-T_{t}(f)(y)\right|^{p} \\
\equiv & \mathrm{E}_{1}+\mathrm{E}_{2}+\mathrm{E}_{3}+\mathrm{E}_{4} .
\end{aligned}
$$

By the Hölder inequality, $L^{p}(\mathcal{X})$-boundedness of $T^{+}$, and (2.1), we have

$$
\mathrm{E}_{1} \lesssim \int_{2 B}\left|f(x)-f_{B}\right|^{p} d \mu(x) \lesssim[\mu(B)]^{1+\alpha p}
$$

On the other hand, using (3.1), (2.1), the Hölder inequality, Lemma 2.4(ii), and $\gamma>\alpha n$, we have that for all $t \in(0,4 r)$ and $x \in B$,

$$
\begin{aligned}
\left|T_{t}\left(f_{2}\right)(x)\right| & \lesssim \int_{(2 B)^{\mathrm{C}}} \frac{1}{V_{t}(x)+V(x, z)}\left(\frac{t}{t+d(x, z)}\right)^{\gamma}\left|f(z)-f_{B}\right| d \mu(z) \\
& \lesssim \sum_{j=1}^{\infty} 2^{-j \gamma} \frac{1}{V_{2^{j-1} r}(x)} \int_{2^{j+1} B}\left[\left|f(z)-f_{2^{j+1} B}\right|+\left|f_{B}-f_{2^{j+1} B}\right|\right] d \mu(z) \\
& \lesssim[\mu(B)]^{\alpha} \sum_{j=1}^{\infty} 2^{-j(\gamma-\alpha n)} \lesssim[\mu(B)]^{\alpha} .
\end{aligned}
$$

This implies that $\mathrm{E}_{3} \lesssim[\mu(B)]^{1+\alpha p}$.

Similarly, by applying (3.1), (2.1), and $\gamma>\alpha n$, we have that for all $x \in B$,

$$
\begin{aligned}
& \left|T_{r}\left(f-f_{B}\right)(x)\right| \\
& \quad \lesssim \int_{\mathcal{X}} \frac{1}{V_{r}(x)+V(x, z)}\left(\frac{r}{r+d(x, z)}\right)^{\gamma}\left|f(z)-f_{B}\right| d \mu(z) \\
& \quad \lesssim \sum_{j=0}^{\infty} 2^{-j \gamma} \frac{1}{V_{2^{j-1} r}(x)} \int_{2^{j+1} B}\left|f(z)-f_{B}\right| d \mu(z) \lesssim[\mu(B)]^{\alpha} .
\end{aligned}
$$


From Lemma 2.4(i), (3.3), $\delta_{2} \geq \alpha n$, and $t<4 r \lesssim \rho\left(x_{0}\right)$, it follows that for all $x \in B$,

$$
\left|f_{B}-T_{t}\left(f_{B}\right)(x)\right|=\left|f_{B}\right|\left|1-T_{t}(1)(x)\right| \lesssim[\mu(B)]^{\alpha}\left(\frac{t}{\rho\left(x_{0}\right)}\right)^{\delta_{2}-\alpha n} \lesssim[\mu(B)]^{\alpha} .
$$

This together with (3.7) implies that

$$
\begin{aligned}
\mathrm{E}_{2} \lesssim \mu(B) \sup _{x, y \in B} \sup _{0<t<4 r}\left\{\left|T_{t}\left(f_{B}\right)(x)-f_{B}\right|^{p}\right. & \\
& \left.\quad+\left|f_{B}-T_{r}\left(f_{B}\right)(y)\right|^{p}+\left|T_{r}\left(f_{B}-f\right)(y)\right|^{p}\right\} \\
& \lesssim[\mu(B)]^{1+\alpha p} .
\end{aligned}
$$

To estimate $\mathrm{E}_{4}$, we first observe that for all $x, y \in B, \rho(x) \sim \rho\left(x_{0}\right) \sim \rho(y)$ (see (3.6)). By this and (3.2), we have that for all $x, y \in B$ and $t \in[4 r, \infty)$,

$$
\left|T_{t}(1)(x)-T_{t}(1)(y)\right| \lesssim\left(\frac{r}{t}\right)^{\beta}
$$

On the other hand, it follows from Lemma 2.4(i) and (2.1) that

$$
\left|f_{B\left(x_{0}, t\right)}\right| \lesssim\left(\frac{\rho\left(x_{0}\right)}{r}\right)^{\alpha n}[\mu(B)]^{\alpha}
$$

Then by these facts and $\alpha n \leq \min \left\{(\beta / 2),\left(\delta_{2} / 2\right)\right\}$, we obtain that for all $t \in[4 r, \infty)$,

$$
\begin{aligned}
\left|T_{t}(1)(x)-T_{t}(1)(y)\right|\left|f_{B\left(x_{0}, t\right)}\right| \\
\lesssim \\
\quad\left(\frac{\rho\left(x_{0}\right)}{r}\right)^{\alpha n}[\mu(B)]^{\alpha}\left|T_{t}(1)(x)-T_{t}(1)(y)\right|^{1 / 2} \\
\quad \times\left[\left|T_{t}(1)(x)-1\right|+\left|1-T_{t}(1)(y)\right|\right]^{1 / 2} \\
\lesssim\left(\frac{\rho\left(x_{0}\right)}{r}\right)^{\alpha n}[\mu(B)]^{\alpha}\left(\frac{r}{\rho\left(x_{0}\right)}\right)^{\min \left\{(\beta / 2),\left(\delta_{2} / 2\right)\right\}} \lesssim[\mu(B)]^{\alpha} .
\end{aligned}
$$

On the other hand, by (3.2), (2.1), the Hölder inequality, Lemma 2.4(ii), $\gamma>\alpha n$, and $\beta \geq \alpha n$, we see that for all $x, y \in B$ and $t \in[4 r, \infty)$,

$$
\begin{aligned}
& \left|T_{t}\left(f-f_{B\left(x_{0}, t\right)}\right)(x)-T_{t}\left(f-f_{B\left(x_{0}, t\right)}\right)(y)\right| \\
& \quad \lesssim \int_{\mathcal{X}}\left(\frac{d(x, y)}{t}\right)^{\beta} \frac{1}{V_{t}(x)+V(x, z)}\left(\frac{t}{t+d(x, z)}\right)^{\gamma}\left|f(z)-f_{B\left(x_{0}, t\right)}\right| d \mu(z)
\end{aligned}
$$




$$
\begin{aligned}
& \lesssim\left(\frac{r}{t}\right)^{\beta} \sum_{j=0}^{\infty} \frac{2^{-j \gamma}}{V_{2^{j-1} t}(x)} \int_{d(x, z)<2^{j} t}\left[\left|f(z)-f_{B\left(x_{0}, 2^{j+1} t\right)}\right|\right. \\
& \left.+\left|f_{B\left(x_{0}, t\right)}-f_{B\left(x_{0}, 2^{j+1} t\right)}\right|\right] d \mu(z) \\
& \lesssim\left(\frac{r}{t}\right)^{\beta} \sum_{j=0}^{\infty} 2^{-j(\gamma-\alpha n)}\left[\mu\left(B\left(x_{0}, t\right)\right)\right]^{\alpha} \lesssim[\mu(B)]^{\alpha} .
\end{aligned}
$$

These inequalities above lead to

$$
\begin{aligned}
\mathrm{E}_{4} \lesssim & \mu(B) \sup _{x, y \in B} \sup _{t \geq 4 r}\left|T_{t}\left(f-f_{B\left(x_{0}, t\right)}\right)(x)-T_{t}\left(f-f_{B\left(x_{0}, t\right)}\right)(y)\right|^{p} \\
& +\mu(B) \sup _{x, y \in B} \sup _{t \geq 4 r}\left[\left|T_{t}(1)(x)-T_{t}(1)(y)\right|\left|f_{B\left(x_{0}, t\right)}\right|\right]^{p} \lesssim[\mu(B)]^{1+\alpha p},
\end{aligned}
$$

which completes the proof of Theorem 3.1.

Now we consider the boundedness of the Poisson semigroup maximal operator. Let $\left\{T_{t}\right\}_{t>0}$ be a family of linear integral operators on $L^{2}(\mathcal{X})$. We always set

$$
P_{t} \equiv \frac{1}{\sqrt{\pi}} \int_{0}^{\infty} \frac{e^{-s}}{\sqrt{s}} T_{t /(2 \sqrt{s})} d s .
$$

For all $f \in L_{\text {loc }}^{1}(\mathcal{X})$, define the Poisson semigroup maximal operator $P^{+}$by

$$
P^{+}(f) \equiv \sup _{t>0}\left|P_{t}(f)\right|
$$

LEMma 3.1. Assume that $\left\{T_{t}\right\}_{t>0}$ satisfies (3.1)-(3.3) with the same constants $\delta_{1}, \delta_{2}, \beta$, and $\gamma$ as there. Then $\left\{P_{t}\right\}_{t>0}$ also satisfies (3.1)-(3.3) with the constants $\delta_{1}, \delta_{2}^{\prime}, \beta^{\prime}$, and $\gamma^{\prime}$, where $\delta_{2}^{\prime} \in(0,1) \cap\left(0, \delta_{2}\right], \beta^{\prime} \in(0,1) \cap(0, \beta]$, and $\gamma^{\prime} \in(0,1) \cap(0, \gamma]$.

Proof. For all $a, s, t \in(0, \infty)$, from the fact that $t+a \leq(1+s)(t / s+a)$, it follows that

$$
\frac{t / s}{t / s+a} \leq\left(1+s^{-1}\right) \frac{t}{t+a} .
$$

On the other hand, from (2.1), we deduce that for all $x, y \in \mathcal{X}$ and $s$, $t \in(0, \infty)$,

$$
V_{t / s}(x)+V(x, y) \sim \mu(B(x, t / s+d(x, y)))
$$




$$
\begin{aligned}
& \gtrsim(1+s)^{-n} \mu(B(x, t+d(x, y))) \\
& \sim(1+s)^{-n}\left[V_{t}(x)+V(x, y)\right] .
\end{aligned}
$$

By (3.1), (3.8), and (3.9), we see that for all $x, y \in \mathcal{X}$,

$$
\begin{aligned}
\left|P_{t}(x, y)\right| & \lesssim \int_{0}^{\infty} e^{-s^{2} / 4} T_{t / s}(x, y) d s \\
& \lesssim \int_{0}^{\infty} e^{-s^{2} / 4} \frac{1}{V_{t / s}(x)+V(x, y)}\left(\frac{t / s}{t / s+d(x, y)}\right)^{\gamma}\left(\frac{\rho(x)}{t / s+\rho(x)}\right)^{\delta_{1}} d s \\
& \lesssim \frac{1}{V_{t}(x)+V(x, y)}\left(\frac{t}{t+d(x, y)}\right)^{\gamma^{\prime}}\left(\frac{\rho(x)}{t+\rho(x)}\right)^{\delta_{1}} \\
& \times \int_{0}^{\infty} e^{-s^{2} / 4}(1+s)^{n+\delta_{1}}\left(1+s^{-\gamma^{\prime}}\right) d s \\
\lesssim & \frac{1}{V_{t}(x)+V(x, y)}\left(\frac{t}{t+d(x, y)}\right)^{\gamma^{\prime}}\left(\frac{\rho(x)}{t+\rho(x)}\right)^{\delta_{1}} .
\end{aligned}
$$

Now we prove that for all $t \in(0, \infty)$ and $x, x^{\prime}, y \in \mathcal{X}$ with $d\left(x, x^{\prime}\right) \leq t / 2$,

(3.10) $\left|P_{t}(x, y)-P_{t}\left(x^{\prime}, y\right)\right| \lesssim\left(\frac{d\left(x, x^{\prime}\right)}{t}\right)^{\beta^{\prime}} \frac{1}{V_{t}(x)+V(x, y)}\left(\frac{t}{t+d(x, y)}\right)^{\gamma^{\prime}}$.

Observe that in this case, $t+d(x, y) \sim t+d\left(x^{\prime}, y\right)$ and $d\left(x, x^{\prime}\right) \leq t /(2 s)$ if and only if $s \leq t /\left[2 d\left(x, x^{\prime}\right)\right]$. Then (3.1) and (3.2) together with (3.8) and (3.9) yield

$$
\begin{aligned}
& \left|P_{t}(x, y)-P_{t}\left(x^{\prime}, y\right)\right| \\
& \lesssim \int_{0}^{\infty} e^{-s^{2} / 4}\left|T_{t / s}(x, y)-T_{t / s}\left(x^{\prime}, y\right)\right| d s \\
& \lesssim \\
& \quad\left[\int_{0}^{t /\left[2 d\left(x, x^{\prime}\right)\right]}\left(\frac{d\left(x, x^{\prime}\right)}{t / s}\right)^{\beta}+\int_{t /\left[2 d\left(x, x^{\prime}\right)\right]}^{\infty}\right] \\
& \quad \times \frac{e^{-s^{2} / 4}}{V_{t / s}(x)+V(x, y)}\left(\frac{t / s}{t / s+d(x, y)}\right)^{\gamma} d s \\
& \lesssim\left[\int_{0}^{t /\left[2 d\left(x, x^{\prime}\right)\right]}(1+s)^{\beta^{\prime}}+\int_{t /\left[2 d\left(x, x^{\prime}\right)\right]}^{\infty} s^{\beta^{\prime}}\right] e^{-s^{2} / 4}(1+s)^{n}\left(1+s^{-\gamma^{\prime}}\right) d s \\
& \quad \times\left(\frac{d\left(x, x^{\prime}\right)}{t}\right)^{\beta^{\prime}} \frac{1}{V_{t}(x)+V(x, y)}\left(\frac{t}{t+d(x, y)}\right)^{\gamma^{\prime}}
\end{aligned}
$$




$$
\lesssim\left(\frac{d\left(x, x^{\prime}\right)}{t}\right)^{\beta^{\prime}} \frac{1}{V_{t}(x)+V(x, y)}\left(\frac{t}{t+d(x, y)}\right)^{\gamma^{\prime}}
$$

which implies (3.10).

On the other hand, by (3.3) and (3.8), we see that for all $x \in \mathcal{X}$ and $t \in(0, \infty)$,

$$
\begin{aligned}
\left|1-P_{t}(1)(x)\right| & \lesssim \int_{0}^{\infty} e^{-s^{2} / 4}\left|1-T_{t / s}(1)(x)\right| d s \\
& \lesssim \int_{0}^{\infty} e^{-s^{2} / 4}\left(\frac{t / s}{t / s+\rho(x)}\right)^{\delta_{2}} d s \\
& \lesssim\left(\frac{t}{t+\rho(x)}\right)^{\delta_{2}^{\prime}} \int_{0}^{\infty} e^{-s^{2} / 4}\left(1+s^{-\delta_{2}^{\prime}}\right) d s \lesssim\left(\frac{t}{t+\rho(x)}\right)^{\delta_{2}^{\prime}} .
\end{aligned}
$$

This finishes the proof of Lemma 3.1.

THEOREM 3.2. Let $\rho$ be an admissible function, let $\left\{T_{t}\right\}_{t>0}$ satisfy (3.1)(3.3) with the same constants $\beta, \gamma, \delta_{1}$, and $\delta_{2}$ as there, and let $\delta_{2}^{\prime}, \beta^{\prime}$, and $\gamma^{\prime}$ be positive constants such that $\delta_{2}^{\prime} \in(0,1) \cap\left(0, \delta_{2}\right], \beta^{\prime} \in(0,1) \cap(0, \beta]$, and $\gamma^{\prime} \in(0,1) \cap(0, \gamma]$. Let $\alpha \in\left(-\infty, \gamma^{\prime} / n\right) \cap\left(-\infty, \min \left\{\beta^{\prime} /(2 n), \delta_{1} / n, \delta_{2}^{\prime} /(2 n)\right\}\right]$, and let $p \in(1, \infty)$. Then there exists a positive constant $C$ such that for all $f \in \mathcal{E}_{\rho}^{\alpha, p}(\mathcal{X}), P^{+}(f) \in \widetilde{\mathcal{E}}_{\rho}^{\alpha, p}(\mathcal{X})$ and

$$
\left\|P^{+}(f)\right\|_{\tilde{\mathcal{E}}_{\rho}^{\alpha, p}(\mathcal{X})} \leq C\|f\|_{\mathcal{E}_{\rho}^{\alpha, p}(\mathcal{X})} .
$$

Proof. Notice that our assumption on $\left\{T_{t}\right\}_{t>0}$ and Lemma 3.1 imply that $\left\{P_{t}\right\}_{t>0}$ satisfies (3.1)-(3.3) with constants $\delta_{1}, \delta_{2}^{\prime}, \gamma^{\prime}$, and $\beta^{\prime}$. By this and an argument similar to the proof of Theorem 3.1, we can prove Theorem 3.2. We omit the details by the similarity. This finishes the proof of Theorem 3.2.

REMARK 3.1. (i) If $\alpha>0$, then by Lemma 2.2(ii), the spaces $\widetilde{\mathcal{E}}_{\rho}^{\alpha, p}(\mathcal{X})$ in Theorems 3.1 and 3.2 are exactly the spaces $\mathcal{E}_{\rho}^{\alpha, p}(\mathcal{X})$. If $\alpha<0$ and $\mathcal{X}$ is an RD-space, then by Remark 2.3(ii) and the fact that the maximal operators are nonnegative, we know that if the space $\widetilde{\mathcal{E}}_{\rho}^{\alpha, p}(\mathcal{X})$ in Theorems 3.1 and 3.2 is replaced by the space $\mathcal{E}_{\rho}^{\alpha, p}(\mathcal{X})$, we obtain the same results.

(ii) Let $\mathcal{X}$ be an RD-space, and let $\rho$ be an admissible function. Assume that there exist constants $C \in(0, \infty), \epsilon_{1} \in(0,1], \epsilon_{2} \in(0, \infty), \delta \in(0,1]$, and $\gamma \in(0, \infty)$ and an $\left(\epsilon_{1}, \epsilon_{2}\right)$-AOTI (approximation of the identity) $\left\{\widetilde{T}_{t}\right\}_{t>0}$ (see, e.g., [12], [32] for the definition of AOTI) with kernels $\left\{\widetilde{T}_{t}(x, y)\right\}_{t>0}$ 
such that for all $t \in(0, \infty)$ and $x, y \in \mathcal{X}$,

$$
\left|T_{t}(x, y)-\widetilde{T}_{t}(x, y)\right| \leq C\left(\frac{t}{t+\rho(x)}\right)^{\delta} \frac{1}{V_{t}(x)+V(x, y)}\left(\frac{t}{t+d(x, y)}\right)^{\gamma}
$$

If $\alpha=0$ and (3.1)-(3.3) were replaced by (3.11), Theorems 3.1 and 3.2 were obtained in [32]. We remark that since for all $x \in \mathcal{X}, \widetilde{T}_{t}(1)(x)=1$ (see [32]), (3.11) implies (3.3) with $\delta_{2}=\delta$.

\section{$\S 4$. Boundedness of the Littlewood-Paley $g$-function}

In this section, we consider the boundedness of certain variants of the Littlewood-Paley $g$-function from $\mathcal{E}_{\rho}^{\alpha, p}(\mathcal{X})$ to $\widetilde{\mathcal{E}}_{\rho}^{\alpha, p}(\mathcal{X})$. The boundedness from $\mathrm{BMO}_{\rho}(\mathcal{X})$ to $\mathrm{BLO}_{\rho}(\mathcal{X})$ where $\mathcal{X}$ is an $\mathrm{RD}$-space of this operator was obtained in [32].

Let $\rho$ be an admissible function on $\mathcal{X}$, and let $\left\{Q_{t}\right\}_{t>0}$ be a family of operators bounded on $L^{2}(\mathcal{X})$ with integral kernels $\left\{Q_{t}(x, y)\right\}_{t>0}$ satisfying that there exist constants $C \in(0, \infty), \delta_{1} \in(0, \infty), \delta_{2} \in(0,1), \beta \in(0,1]$, and $\gamma \in(0, \infty)$ such that for all $t \in(0, \infty)$ and $x, x^{\prime}, y \in \mathcal{X}$ with $d\left(x, x^{\prime}\right) \leq(t / 2)$,

$$
\begin{gathered}
(Q)_{\mathrm{i}} \quad\left|Q_{t}(x, y)\right| \leq C \frac{1}{V_{t}(x)+V(x, y)}\left(\frac{t}{t+d(x, y)}\right)^{\gamma}\left(\frac{\rho(x)}{t+\rho(x)}\right)^{\delta_{1}} \\
(Q)_{\mathrm{ii}}\left|Q_{t}(x, y)-Q_{t}\left(x^{\prime}, y\right)\right| \leq \\
\times\left(\frac{d\left(x, x^{\prime}\right)}{t+d(x, y)}\right)^{\beta} \frac{1}{V_{t}(x)+V(x, y)} \\
\times\left(\frac{t}{t+d(x, y)}\right)^{\gamma} \\
(Q)_{\mathrm{iii}}\left|\int_{\mathcal{X}} Q_{t}(x, y) d \mu(y)\right| \leq C\left(\frac{t}{t+\rho(x)}\right)^{\delta_{2}}
\end{gathered}
$$

For all $f \in L_{\text {loc }}^{1}(\mathcal{X})$ and $x \in \mathcal{X}$, define the Littlewood-Paley $g$-function by

$$
g(f)(x) \equiv\left(\int_{0}^{\infty}\left|Q_{t}(f)(x)\right|^{2} \frac{d t}{t}\right)^{1 / 2}
$$

Lemma 4.1. Let $\alpha \in\left(-\infty, \min \left\{\gamma / n, \delta_{2} / n\right\}\right)$, let $p \in(1, \infty)$, and let $\rho$ be an admissible function on $\mathcal{X}$. Then there exists a positive constant $C$ such that for all $f \in \mathcal{E}_{\rho}^{\alpha, p}(\mathcal{X})$,

(i) for all $x \in \mathcal{X}$ and $t>0$,

$$
\left|Q_{t}(f)(x)\right| \leq C\left(\frac{\rho(x)}{t+\rho(x)}\right)^{\delta_{1}}[\mu(B(x, t))]^{\alpha}\|f\|_{\mathcal{E}_{\rho}^{\alpha, p}(\mathcal{X})} ;
$$


(ii) for all $x, y \in \mathcal{X}$ and $t \geq 2 d(x, y)$,

$$
\begin{aligned}
& \left|Q_{t}(f)(x)-Q_{t}(f)(y)\right| \\
& \quad \leq \begin{cases}C\left(\frac{d(x, y)}{t}\right)^{\beta}\left(1+\frac{\rho(x)}{t}\right)^{\alpha n}[\mu(B(x, t))]^{\alpha}\|f\|_{\mathcal{E}_{\rho}^{\alpha, p}(\mathcal{X})}, & \alpha>0, \\
C\left(\frac{d(x, y)}{t}\right)^{\beta}\left(1+\log \frac{\rho(x)}{t}\right)[\mu(B(x, t))]^{\alpha}\|f\|_{\mathcal{E}_{\rho}^{\alpha, p}(\mathcal{X})}, & \alpha \leq 0 .\end{cases}
\end{aligned}
$$

Proof. By the homogeneity of $\|\cdot\|_{\mathcal{E}_{\rho}^{\alpha, p}(\mathcal{X})}$, we may assume that $f \in \mathcal{E}_{\rho}^{\alpha, p}(\mathcal{X})$ and that $\|f\|_{\mathcal{E}_{\rho}^{\alpha, p}(\mathcal{X})}=1$. By $(Q)_{\mathrm{i}},(4.2),(2.1), \gamma>\alpha n$, and the Hölder inequality, we have that for all $x \in \mathcal{X}$ and $t \geq \rho(x)$,

$$
\begin{aligned}
\left|Q_{t}(f)(x)\right| \lesssim & \int_{\mathcal{X}} \frac{1}{V_{t}(x)+V(x, y)}\left(\frac{t}{t+d(x, y)}\right)^{\gamma} \\
& \times\left(\frac{\rho(x)}{t+\rho(x)}\right)^{\delta_{1}}|f(y)| d \mu(y) \\
\lesssim & \left(\frac{\rho(x)}{t+\rho(x)}\right)^{\delta_{1}} \sum_{j=0}^{\infty} 2^{-j \gamma} \frac{1}{V_{2^{j-1} t}(x)} \int_{d(x, y)<2^{j} t}|f(y)| d \mu(y) \\
\lesssim & \left(\frac{\rho(x)}{t+\rho(x)}\right)^{\delta_{1}} \sum_{j=0}^{\infty} 2^{-j \gamma}\left[\mu\left(B\left(x, 2^{j} t\right)\right)\right]^{\alpha} \\
\lesssim & \left(\frac{\rho(x)}{t+\rho(x)}\right)^{\delta_{1}}[\mu(B(x, t))]^{\alpha} \sum_{j=0}^{\infty} \max \left\{2^{-j(\gamma-\alpha n)}, 2^{-j \gamma}\right\} \\
\lesssim & \left(\frac{\rho(x)}{t+\rho(x)}\right)^{\delta_{1}}[\mu(B(x, t))]^{\alpha}
\end{aligned}
$$

Let $x \in \mathcal{X}$, and let $t<\rho(x)$. In this case, $t+\rho(x) \sim \rho(x)$. Using $\gamma>\alpha n$, $(Q)_{\mathrm{i}},(2.1)$, Lemma 2.4(ii), and the Hölder inequality, we have

$$
\begin{gathered}
\left|Q_{t}\left(f-f_{B(x, t)}\right)(x)\right| \\
\lesssim \sum_{j=0}^{\infty} 2^{-j \gamma} \frac{1}{V_{2^{j-1} t}(x)} \int_{d(x, y)<2^{j} t}\left|f(y)-f_{B(x, t)}\right| d \mu(y) \\
\lesssim \sum_{j=0}^{\infty} 2^{-j \gamma}\left\{\frac{1}{V_{2^{j} t}(x)} \int_{d(x, y)<2^{j} t}\left|f(y)-f_{B\left(x, 2^{j} t\right)}\right| d \mu(y)\right. \\
\left.+\left|f_{B\left(x, 2^{j} t\right)}-f_{B(x, t)}\right|\right\}
\end{gathered}
$$




$$
\lesssim[\mu(B(x, t))]^{\alpha} \sum_{j=0}^{\infty} 2^{-j \gamma} \max \left\{2^{j \max \{\alpha n, 0\}}, j+1\right\} \lesssim[\mu(B(x, t))]^{\alpha} .
$$

On the other hand, from $(Q)_{\mathrm{iii}}$, Lemma 2.4(i), $t<\rho(x)$, and $\delta_{2}>\alpha n$, we deduce that

$$
\begin{aligned}
\left|Q_{t}\left(f_{B(x, t)}\right)(x)\right| \lesssim & {[\mu(B(x, t))]^{\alpha}\left(\frac{t}{t+\rho(x)}\right)^{\delta_{2}} } \\
& \times \max \left\{1+\log \frac{\rho(x)}{t},\left(\frac{\rho(x)}{t}\right)^{\max \{\alpha n, 0\}}\right\} \\
\lesssim & {[\mu(B(x, t))]^{\alpha}\left(\frac{\rho(x)}{t+\rho(x)}\right)^{\delta_{1}} . }
\end{aligned}
$$

This gives (i).

To show (ii), by $(Q)_{\text {ii }}$, we see that for all $x, y \in \mathcal{X}$ and $t \geq 2 d(x, y)$,

$$
\begin{aligned}
& \left|Q_{t}(f)(x)-Q_{t}(f)(y)\right| \\
& \quad \lesssim \int_{\mathcal{X}}\left(\frac{d(x, y)}{t+d(x, z)}\right)^{\beta} \frac{1}{V_{t}(x)+V(x, z)}\left(\frac{t}{t+d(x, z)}\right)^{\gamma}|f(z)| d \mu(z) \\
& \quad \lesssim\left(\frac{d(x, y)}{t}\right)^{\beta} \sum_{j=0}^{\infty} 2^{-j \gamma} \frac{1}{V_{2^{j-1} t}(x)} \int_{d(x, z)<2^{j} t}|f(z)| d \mu(z) .
\end{aligned}
$$

Now we consider the following two cases.

Case (i). $\alpha \in(0, \infty)$. In this case, if $t \geq \rho(x)$, by $\gamma>\alpha n$, the Hölder inequality, (4.3), and (2.1), we have

$$
\begin{aligned}
\left|Q_{t}(f)(x)-Q_{t}(f)(y)\right| & \lesssim\left(\frac{d(x, y)}{t}\right)^{\beta} \sum_{j=0}^{\infty} 2^{-j \gamma}\left[\mu\left(B\left(x, 2^{j} t\right)\right)\right]^{\alpha} \\
& \lesssim\left(\frac{d(x, y)}{t}\right)^{\beta}[\mu(B(x, t))]^{\alpha} .
\end{aligned}
$$

Assume that $t<\rho(x)$. Let $N_{1} \in \mathbb{N}$ such that $2^{N_{1}-1} t<\rho(x) \leq 2^{N_{1}} t$. From the Hölder inequality and (2.1), it follows that

$$
\begin{aligned}
& \sum_{j=N_{1}}^{\infty} 2^{-j \gamma} \frac{1}{V_{2^{j-1} t}(x)} \int_{d(x, z)<2^{j} t}|f(z)| d \mu(z) \\
& \lesssim \sum_{j=N_{1}}^{\infty} 2^{-j \gamma}\left[\mu\left(B\left(x, 2^{j} t\right)\right)\right]^{\alpha} \lesssim[\mu(B(x, t))]^{\alpha} .
\end{aligned}
$$


By the Hölder inequality, (2.1), and Lemma 2.4(i), we see that for all $j \in$ $\left\{0,1, \ldots, N_{1}-1\right\}$,

$$
\frac{1}{V_{2^{j-1} t}(x)} \int_{d(x, z)<2^{j} t}|f(z)| d \mu(z) \lesssim\left(\frac{\rho(x)}{t}\right)^{\alpha n}[\mu(B(x, t))]^{\alpha} .
$$

This together with $\gamma>\alpha$ gives us

$$
\sum_{j=0}^{N_{1}-1} 2^{-j \gamma} \frac{1}{V_{2^{j-1} t}(x)} \int_{d(x, z)<2^{j} t}|f(z)| d \mu(z) \lesssim\left(\frac{\rho(x)}{t}\right)^{\alpha n}[\mu(B(x, t))]^{\alpha} .
$$

Combining this and (4.3)-(4.5) leads to that for all $x, y \in \mathcal{X}$ and $t \geq 2 d(x, y)$,

$$
\left|Q_{t}(f)(x)-Q_{t}(f)(y)\right| \lesssim\left(1+\frac{\rho(x)}{t}\right)^{\alpha n}[\mu(B(x, t))]^{\alpha} .
$$

Case (ii). $\alpha \in(-\infty, 0]$. If $t \geq \rho(x)$, then (4.3) yields that

$$
\left|Q_{t}(f)(x)-Q_{t}(f)(y)\right| \lesssim\left(\frac{d(x, y)}{t}\right)^{\beta}[\mu(B(x, t))]^{\alpha} .
$$

Let $t<\rho(x)$, and let $N_{1}$ be the integer as in Case (i). Then by (4.3), (2.1), Lemma 2.4(i), and the Hölder inequality, we have

$$
\begin{aligned}
& \left|Q_{t}(f)(x)-Q_{t}(f)(y)\right| \\
& \quad \lesssim\left(\frac{d(x, y)}{t}\right)^{\beta}\left\{\sum_{j=0}^{N_{1}-1} 2^{-j \gamma} \frac{1}{V_{2^{j-1} t}(x)} \int_{d(x, z)<2^{j} t}|f(z)| d \mu(z)+\sum_{j=N_{1}}^{\infty} \cdots\right\} \\
& \quad \lesssim\left(\frac{d(x, y)}{t}\right)^{\beta}\left\{\sum_{j=0}^{N_{1}-1} 2^{-j \gamma}\left(1+\log \frac{\rho(x)}{t}\right)+\sum_{j=N_{1}}^{\infty} 2^{-j \gamma}\right\}[\mu(B(x, t))]^{\alpha} \\
& \quad \lesssim\left(\frac{d(x, y)}{t}\right)^{\beta}\left(1+\log \frac{\rho(x)}{t}\right)[\mu(B(x, t))]^{\alpha},
\end{aligned}
$$

which implies (ii) and then completes the proof of Lemma 4.1.

TheOREM 4.1. Let $p \in(1, \infty)$, let $\rho$ be an admissible function on $\mathcal{X}$, let $g$ be as in (4.1), and let

$$
\alpha \in(-\infty, \beta /(3 n)] \cap\left(-\infty, \min \left\{\gamma / n, \delta_{1} / n, \delta_{2} /(3 n)\right\}\right) .
$$

If $g(\cdot)$ is bounded on $L^{p}(\mathcal{X})$, then there exists a positive constant $C$ such that for all $f \in \mathcal{E}_{\rho}^{\alpha, p}(\mathcal{X}),[g(f)]^{2} \in \widetilde{\mathcal{E}}_{\rho}^{2 \alpha, p / 2}(\mathcal{X})$ and $\left\|[g(f)]^{2}\right\|_{\widetilde{\mathcal{E}}_{\rho}^{2 \alpha, p / 2}(\mathcal{X})} \leq$ $C\|f\|_{\mathcal{E}_{\rho}^{\alpha, p}(\mathcal{X})}^{2}$. 
Proof. By similarity, we prove only the case when $\alpha>0$. Let $f \in \mathcal{E}_{\rho}^{\alpha, p}(\mathcal{X})$. By the homogeneity of $\|\cdot\|_{\mathcal{E}_{\rho}^{\alpha, p}(\mathcal{X})}$ and $\|\cdot\|_{\widetilde{\mathcal{E}}_{\rho}^{\alpha, p}(\mathcal{X})}$, we may assume that $\|f\|_{\mathcal{E}_{\rho}^{\alpha, p}(\mathcal{X})}=1$. For all balls $B \equiv B\left(x_{0}, r\right) \in \mathcal{D}_{\rho}$, we need to prove that

$$
\int_{B}[g(f)(x)]^{p} d \mu(x) \lesssim[\mu(B)]^{1+\alpha p} .
$$

For all $x \in \mathcal{X}$, write

$$
\begin{aligned}
{[g(f)(x)]^{2} } & =\int_{0}^{8 r}\left|Q_{t}(f)(x)\right|^{2} \frac{d t}{t}+\int_{8 r}^{\infty}\left|Q_{t}(f)(x)\right|^{2} \frac{d t}{t} \\
& \equiv\left[g_{1}(f)(x)\right]^{2}+\left[g_{2}(f)(x)\right]^{2}
\end{aligned}
$$

By the $L^{p}(\mathcal{X})$-boundedness of $g$ and (2.1), we have

$$
\int_{B}\left[g_{1}\left(f \chi_{2 B}\right)(x)\right]^{p} d \mu(x) \lesssim \int_{2 B}|f(x)|^{p} d \mu(x) \lesssim[\mu(B)]^{1+\alpha p} .
$$

By $(Q)_{\mathrm{i}}, \gamma>\alpha n,(2.1)$, and the Hölder inequality, we have that for all $x \in B$ and $t<8 r$,

$$
\begin{aligned}
\left|Q_{t}\left(f \chi_{(2 B)^{\complement}}\right)(x)\right| & \lesssim \int_{(2 B)^{\complement}} \frac{1}{V_{t}(x)+V(x, y)}\left(\frac{t}{t+d(x, y)}\right)^{\gamma}|f(y)| d \mu(y) \\
& \lesssim\left(\frac{t}{r}\right)^{\gamma} \sum_{j=1}^{\infty} 2^{-j \gamma} \frac{1}{\mu\left(2^{j+1} B\right)} \int_{2^{j+1} B}|f(y)| d \mu(y) \\
& \lesssim\left(\frac{t}{r}\right)^{\gamma}[\mu(B)]^{\alpha} \sum_{j=1}^{\infty} 2^{-j(\gamma-\alpha n)} \lesssim\left(\frac{t}{r}\right)^{\gamma}[\mu(B)]^{\alpha}
\end{aligned}
$$

From this, it follows that

$$
\begin{aligned}
& \int_{B}\left[g_{1}\left(f \chi_{(2 B)^{\complement}}\right)(x)\right]^{p} d \mu(x) \\
& \quad \lesssim\left(\int_{0}^{8 r}\left(\frac{t}{r}\right)^{2 \gamma} \frac{d t}{t}\right)^{p / 2}[\mu(B)]^{1+\alpha p} \lesssim[\mu(B)]^{1+\alpha p} .
\end{aligned}
$$

Combining (4.7) and (4.8) leads to

$$
\int_{B}\left[g_{1}(f)(x)\right]^{p} d \mu(x) \lesssim[\mu(B)]^{1+\alpha p} .
$$


Applying [33, Lemma 2.1(ii) and 2.1(iii)], we have that for all $x, y \in \mathcal{X}$,

$$
\frac{1}{\rho(x)} \gtrsim \frac{1}{\rho(y)}\left(1+\frac{d(x, y)}{\rho(y)}\right)^{-k_{0} /\left(1+k_{0}\right)},
$$

where $k_{0}$ is as in Definition 2.2. By this fact, we obtain that for all $x \in B$ and $t \geq 8 r$,

$$
\frac{1}{\rho(x)} \gtrsim \frac{1}{\rho\left(x_{0}\right)}\left(1+\frac{r}{\rho\left(x_{0}\right)}\right)^{-k_{0} /\left(1+k_{0}\right)} \gtrsim \frac{1}{\rho\left(x_{0}\right)}\left(\frac{r}{\rho\left(x_{0}\right)}\right)^{-k_{0} /\left(1+k_{0}\right)} .
$$

From this, Lemma 4.1(i), and (2.1), we deduce that for all $x \in B$,

$$
\begin{aligned}
\left|Q_{t}(f)(x)\right| & \lesssim\left(\frac{\rho(x)}{t}\right)^{\delta_{1}}[\mu(B(x, t))]^{\alpha} \\
& \lesssim\left(\frac{\rho\left(x_{0}\right)}{t}\right)^{\delta_{1}}\left(\frac{r}{\rho\left(x_{0}\right)}\right)^{\delta_{1}\left(k_{0} /\left(1+k_{0}\right)\right)}\left(\frac{t}{r}\right)^{\alpha n}[\mu(B)]^{\alpha},
\end{aligned}
$$

which together with the assumption that $\delta_{1}>\alpha n$ implies that

$$
\begin{aligned}
& \int_{B}\left[g_{2}(f)(x)\right]^{p} d \mu(x) \\
& \quad \lesssim[\mu(B)]^{1+\alpha p}\left(\frac{r}{\rho\left(x_{0}\right)}\right)^{p \delta_{1}\left(k_{0} /\left(1+k_{0}\right)\right)}\left\{\int_{8 r}^{\infty}\left(\frac{\rho\left(x_{0}\right)}{t}\right)^{2 \delta_{1}}\left(\frac{t}{r}\right)^{2 \alpha n} \frac{d t}{t}\right\}^{p / 2} \\
& \quad \lesssim[\mu(B)]^{1+\alpha p}\left(\frac{r}{\rho\left(x_{0}\right)}\right)^{p \delta_{1}\left(k_{0} /\left(1+k_{0}\right)\right)}\left(\frac{\rho\left(x_{0}\right)}{r}\right)^{p \delta_{1}} \\
& \quad \lesssim[\mu(B)]^{1+\alpha p} .
\end{aligned}
$$

This together with (4.9) gives (4.6). Moreover, it follows from (4.6) that $g(f)(x)<\infty$ for almost every $x \in \mathcal{X}$.

Now we assume that $B \equiv B\left(x_{0}, r\right) \notin \mathcal{D}_{\rho}$. We need to prove that

$$
\int_{B}\left\{[g(f)(x)]^{2}-\underset{B}{\operatorname{essinf}}[g(f)]^{2}\right\}^{p / 2} d \mu(x) \lesssim[\mu(B)]^{1+\alpha p} .
$$

To this end, write

$$
\begin{aligned}
{[g(f)(x)]^{2} } & =\int_{0}^{8 r}\left|Q_{t}(f)(x)\right|^{2} \frac{d t}{t}+\int_{8 r}^{8 \rho\left(x_{0}\right)} \cdots+\int_{8 \rho\left(x_{0}\right)}^{\infty} \cdots \\
& \equiv\left[g_{r}(f)(x)\right]^{2}+\left[g_{r, \rho\left(x_{0}\right)}(f)(x)\right]^{2}+\left[g_{\rho\left(x_{0}\right), \infty}(f)(x)\right]^{2}
\end{aligned}
$$


Then

$$
\begin{aligned}
\int_{B}\{ & {\left.[g(f)(x)]^{2}-\underset{B}{\operatorname{essinf}}[g(f)]^{2}\right\}^{p / 2} d \mu(x) } \\
\lesssim & \int_{B}\left[g_{r}(f)(x)\right]^{p} d \mu(x) \\
& +\int_{B}\left\{\left[g_{r, \rho\left(x_{0}\right)}(f)(x)\right]^{2}-\underset{B}{\operatorname{essinf}}\left[g_{r, \rho\left(x_{0}\right)}(f)\right]^{2}\right\}^{p / 2} d \mu(x) \\
& +\int_{B}\left\{\left[g_{\rho\left(x_{0}\right), \infty}(f)(x)\right]^{2}-\underset{B}{\operatorname{essinf}}\left[g_{\rho\left(x_{0}\right), \infty}(f)\right]^{2}\right\}^{p / 2} d \mu(x) \\
\lesssim & \int_{B}\left[g_{r}(f)(x)\right]^{p} d \mu(x)+\mu(B) \sup _{x, y \in B}\left|\left[g_{r, \rho\left(x_{0}\right)}(f)(x)\right]^{2}-\left[g_{r, \rho\left(x_{0}\right)}(f)(y)\right]^{2}\right|^{p / 2} \\
& +\mu(B) \sup _{x, y \in B}\left|\left[g_{\rho\left(x_{0}\right), \infty}(f)(x)\right]^{2}-\left[g_{\rho\left(x_{0}\right), \infty}(f)(y)\right]^{2}\right|^{p / 2} \equiv \mathrm{I}_{1}+\mathrm{I}_{2}+\mathrm{I}_{3} .
\end{aligned}
$$

Write $f=\left(f-f_{B}\right) \chi_{2 B}+\left(f-f_{B}\right) \chi_{(2 B)^{\complement}}+f_{B} \equiv f_{1}+f_{2}+f_{B}$. By the $L^{p}(\mathcal{X})$-boundedness of $g(\cdot)$ and $(2.1)$, we have

$$
\int_{B}\left[g_{r}\left(f_{1}\right)(x)\right]^{p} d \mu(x) \lesssim \int_{2 B}\left|f(x)-f_{B}\right|^{p} d \mu(x) \lesssim[\mu(B)]^{1+\alpha p} .
$$

Using $(Q)_{\mathrm{i}},(2.1)$, the Hölder inequality, Lemma 2.4(ii), and $\gamma>\alpha n$, we obtain that for all $x \in B$,

$$
\begin{aligned}
& \left|Q_{t}\left(f_{2}\right)(x)\right| \\
& \quad \lesssim \int_{(2 B)^{\complement}} \frac{1}{V_{t}(x)+V(x, y)}\left(\frac{t}{t+d(x, y)}\right)^{\gamma}\left|f(y)-f_{B}\right| d \mu(y) \\
& \quad \lesssim\left(\frac{t}{r}\right)^{\gamma} \sum_{j=1}^{\infty} 2^{-j \gamma} \frac{1}{\mu\left(2^{j+1} B\right)} \int_{2^{j+1} B}\left[\left|f(y)-f_{2^{j+1} B}\right|+\left|f_{2^{j+1} B}-f_{B}\right|\right] d \mu(y) \\
& \quad \lesssim\left(\frac{t}{r}\right)^{\gamma}[\mu(B)]^{\alpha} \sum_{j=1}^{\infty} 2^{-j(\gamma-\alpha n)} \lesssim\left(\frac{t}{r}\right)^{\gamma}[\mu(B)]^{\alpha},
\end{aligned}
$$

from which it follows that

$$
\int_{B}\left[g_{r}\left(f_{2}\right)(x)\right]^{p} d \mu(x) \lesssim[\mu(B)]^{1+\alpha p}\left(\int_{0}^{8 r}\left(\frac{t}{r}\right)^{2 \gamma} \frac{d t}{t}\right)^{p / 2} \lesssim[\mu(B)]^{1+\alpha p}
$$


Recall that for all $x \in B, \rho(x) \sim \rho\left(x_{0}\right)$ (see (3.6)). By this, $(Q)_{\text {iii }}$, and Lemma 2.4(i), we have that for all $x \in B$,

$$
\left|Q_{t}\left(f_{B}\right)(x)\right| \lesssim\left(\frac{t}{t+\rho(x)}\right)^{\delta_{2}}\left|f_{B}\right| \lesssim\left(\frac{t}{\rho\left(x_{0}\right)}\right)^{\delta_{2}}\left(\frac{\rho\left(x_{0}\right)}{r}\right)^{\alpha n}[\mu(B)]^{\alpha}
$$

This together with $\delta_{2}>3 \alpha n$ and $r<\rho\left(x_{0}\right)$ implies that

$$
\begin{aligned}
& \int_{B}\left[g_{r}\left(f_{B}\right)(x)\right]^{p} d \mu(x) \\
& \quad \lesssim[\mu(B)]^{1+\alpha p}\left(\frac{\rho\left(x_{0}\right)}{r}\right)^{\alpha p n}\left(\int_{0}^{8 r}\left(\frac{t}{\rho\left(x_{0}\right)}\right)^{2 \delta_{2}} \frac{d t}{t}\right)^{p / 2} \\
& \quad \lesssim[\mu(B)]^{1+\alpha p} .
\end{aligned}
$$

Combining this, (4.11), and (4.12) yields $\mathrm{I}_{1} \lesssim[\mu(B)]^{1+\alpha p}$.

Since $\gamma>\alpha n$, by Lemma 4.1, (2.1), and $\rho\left(x_{0}\right) \sim \rho(x)$ for all $x \in B$, we have that for all $x, y \in B$ and $t \in\left[8 \rho\left(x_{0}\right), \infty\right)$,

$$
\left|Q_{t}(f)(x)-Q_{t}(f)(y)\right| \lesssim\left(\frac{d(x, y)}{t}\right)^{\beta}[\mu(B(x, t))]^{\alpha} \lesssim\left(\frac{r}{t}\right)^{\beta-\alpha n}[\mu(B)]^{\alpha},
$$

and

$$
\left|Q_{t}(f)(x)\right| \lesssim\left(\frac{\rho\left(x_{0}\right)}{t}\right)^{\delta_{1}}[\mu(B(x, t))]^{\alpha} \lesssim\left(\frac{\rho\left(x_{0}\right)}{t}\right)^{\delta_{1}}\left(\frac{t}{r}\right)^{\alpha n}[\mu(B)]^{\alpha} .
$$

By these inequalities and $\beta \geq 3 \alpha n$, we see that for all $x, y \in B$,

$$
\begin{aligned}
& {\left[g_{\rho\left(x_{0}\right), \infty}(f)(x)\right]^{2}-\left[g_{\rho\left(x_{0}\right), \infty}(f)(y)\right]^{2}} \\
& \quad \leq \int_{8 \rho\left(x_{0}\right)}^{\infty}\left|Q_{t}(f)(x)+Q_{t}(f)(y)\right|\left|Q_{t}(f)(x)-Q_{t}(f)(y)\right| \frac{d t}{t} \\
& \quad \leq \int_{8 \rho\left(x_{0}\right)}^{\infty}\left(\frac{\rho\left(x_{0}\right)}{t}\right)^{\delta_{1}}\left(\frac{r}{t}\right)^{\beta-2 \alpha n}[\mu(B)]^{2 \alpha} \frac{d t}{t} \lesssim[\mu(B)]^{2 \alpha}
\end{aligned}
$$

which implies that $\mathrm{I}_{3} \lesssim[\mu(B)]^{1+\alpha p}$.

By Lemma 4.1(i), (2.1), and the fact that for all $x \in B, \rho\left(x_{0}\right) \sim \rho(x)$, we have that for all $t \in\left[8 r, 8 \rho\left(x_{0}\right)\right)$ and $x \in B$,

$$
\left|Q_{t}(f)(x)\right| \lesssim[\mu(B(x, t))]^{\alpha} \lesssim\left(\frac{t}{r}\right)^{\alpha n}[\mu(B)]^{\alpha}
$$


Thus the fact that $\beta \geq 3 \alpha n$ implies that for all $x, y \in B$,

$$
\begin{aligned}
& {\left[g_{r, \rho\left(x_{0}\right)}(f)(x)\right]^{2}-\left[g_{r, \rho\left(x_{0}\right)}(f)(y)\right]^{2}} \\
& \quad \leq \int_{8 r}^{8 \rho\left(x_{0}\right)}\left|Q_{t}(f)(x)+Q_{t}(f)(y)\right|\left|Q_{t}(f)(x)-Q_{t}(f)(y)\right| \frac{d t}{t} \\
& \quad \lesssim[\mu(B)]^{\alpha} \int_{8 r}^{8 \rho\left(x_{0}\right)}\left(\frac{t}{r}\right)^{\alpha n}\left|Q_{t}(f)(x)-Q_{t}(f)(y)\right| \frac{d t}{t} .
\end{aligned}
$$

Let $t \in\left[8 r, 8 \rho\left(x_{0}\right)\right)$, and let $x, y \in B$. We write

$$
\begin{aligned}
& \left|Q_{t}(f)(x)-Q_{t}(f)(y)\right| \\
& \leq\left|\int_{\mathcal{X}}\left[Q_{t}(x, z)-Q_{t}(y, z)\right]\left[f(z)-f_{B}\right] d \mu(z)\right| \\
& \quad+\left|f_{B}\right|\left|\int_{\mathcal{X}}\left[Q_{t}(x, z)-Q_{t}(y, z)\right] d \mu(z)\right| \\
& \equiv \mathrm{H}_{1}+\mathrm{H}_{2} .
\end{aligned}
$$

By $(Q)_{\mathrm{ii}}, t \in\left[8 r, 8 \rho\left(x_{0}\right)\right),(2.1)$, and Lemma 2.4(ii), we see that for all $x \in B$,

$$
\begin{aligned}
\mathrm{H}_{1} \lesssim \int_{\mathcal{X}}\left(\frac{d(x, y)}{t+d(x, z)}\right)^{\beta} \frac{1}{V_{t}(x)+V(x, z)}\left(\frac{t}{t+d(x, z)}\right)^{\gamma}\left|f(z)-f_{B}\right| d \mu(z) \\
\lesssim \sum_{j=0}^{\infty} \frac{r^{\beta} t^{\gamma}}{\left(t+2^{j-1} r\right)^{\beta+\gamma}} \frac{1}{\mu\left(2^{j+1} B\right)} \\
\quad \times \int_{2^{j+1} B}\left\{\left|f(z)-f_{2^{j+1} B}\right|+\left|f_{2^{j+1} B}-f_{B}\right|\right\} d \mu(z) \\
\lesssim \sum_{j=0}^{\infty} \frac{r^{\beta} t^{\gamma}}{\left(t+2^{j} r\right)^{\beta+\gamma}} 2^{j \alpha n}[\mu(B)]^{\alpha} .
\end{aligned}
$$

From this, we deduce that

$$
\begin{aligned}
& \int_{8 r}^{8 \rho\left(x_{0}\right)}\left(\frac{t}{r}\right)^{\alpha n} \mathrm{H}_{1} \frac{d t}{t} \\
& \quad \lesssim[\mu(B)]^{\alpha} \sum_{j=0}^{\infty} 2^{j \alpha n} \int_{8 r}^{8 \rho\left(x_{0}\right)}\left(\frac{r}{t}\right)^{\beta-\alpha n} \frac{t^{\gamma+\beta-1}}{\left(t+2^{j} r\right)^{\beta+\gamma}} d t \\
& \quad \lesssim[\mu(B)]^{\alpha}
\end{aligned}
$$


By Lemma 2.4(i), $(Q)_{\text {iii }}, \beta \geq 3 \alpha n, \delta_{2}>3 \alpha n$, and the fact that for all $z \in B, \rho\left(x_{0}\right) \sim \rho(z)$, we have that for $\mu$ almost every $x, y \in B$,

$$
\begin{aligned}
& \int_{8 r}^{8 \rho\left(x_{0}\right)}\left(\frac{t}{r}\right)^{\alpha n} \mathrm{H}_{2} \frac{d t}{t} \\
& \leq \int_{8 r}^{8 \rho\left(x_{0}\right)}\left(\frac{t}{r}\right)^{\alpha n}\left(\frac{\rho\left(x_{0}\right)}{r}\right)^{\alpha n}[\mu(B)]^{\alpha}\left|Q_{t}(1)(x)-Q_{t}(1)(y)\right|^{2 / 3} \\
& \quad \times\left(\frac{t}{\rho\left(x_{0}\right)}\right)^{\delta_{2} / 3} \frac{d t}{t} \\
& \lesssim \int_{8 r}^{8 \rho\left(x_{0}\right)}\left(\frac{\rho\left(x_{0}\right)}{r}\right)^{\alpha n}[\mu(B)]^{\alpha}\left(\frac{r}{t}\right)^{\beta / 3}\left(\frac{t}{\rho\left(x_{0}\right)}\right)^{\delta_{2} / 3} \frac{d t}{t} \\
& \lesssim \int_{8 r}^{\rho\left(x_{0}\right)}[\mu(B)]^{\alpha}\left(\frac{t}{\rho\left(x_{0}\right)}\right)^{\left(\delta_{2} / 3\right)-\alpha n} \frac{d t}{t} \lesssim[\mu(B)]^{\alpha} .
\end{aligned}
$$

This finishes the proof of Theorem 4.1.

As a consequence of Theorem 4.1, we have the following conclusion.

Corollary 4.1. With the same assumptions as in Theorem 4.1, there exists a positive constant $C$ such for all $f \in \mathcal{E}_{\rho}^{\alpha, p}(\mathcal{X}), g(f) \in \widetilde{\mathcal{E}}_{\rho}^{\alpha, p}(\mathcal{X})$ and $\|g(f)\|_{\widetilde{\mathcal{E}}_{\rho}^{\alpha, p}(\mathcal{X})} \leq C\|f\|_{\mathcal{E}_{\rho}^{\alpha, p}(\mathcal{X})}$.

Proof. Since

$$
0 \leq g(f)-\underset{B}{\operatorname{essinf}} g(f) \leq\left\{[g(f)]^{2}-\underset{B}{\operatorname{essinf}}[g(f)]^{2}\right\}^{1 / 2},
$$

applying (4.10), we have that for all balls $B \notin \mathcal{D}_{\rho}$,

$$
\begin{aligned}
& \left\{\frac{1}{[\mu(B)]^{1+\alpha p}} \int_{B}[g(f)(x)-\underset{B}{\operatorname{essinf}} g(f)]^{p} d \mu(x)\right\}^{1 / p} \\
& \quad \lesssim\left\{\frac{1}{[\mu(B)]^{1+\alpha p}} \int_{B}\left\{[g(f)(x)]^{2}-\underset{B}{\operatorname{essinf}}[g(f)]^{2}\right\}^{p / 2} d \mu(x)\right\}^{1 / p} \\
& \quad \lesssim\|f\|_{\mathcal{E}_{\rho}^{\alpha, p}(\mathcal{X})} .
\end{aligned}
$$

On the other hand, by (4.6), we obtain that for all balls $B \in \mathcal{D}_{\rho}$,

$$
\left\{\frac{1}{[\mu(B)]^{1+\alpha p}} \int_{B}[g(f)(x)]^{p} d \mu(x)\right\}^{1 / p} \lesssim\|f\|_{\mathcal{E}_{\rho}^{\alpha, p}(\mathcal{X})}
$$

which together with (4.13) completes the proof of Corollary 4.1. 
REMARK 4.1. (i) If $\alpha=0$ and $\mathcal{X}$ is an RD-space, Theorem 4.1 and Corollary 4.1 were already obtained in [32].

(ii) We point out that Remark 3.1(i) is also suitable to Theorem 4.1 and Corollary 4.1.

\section{$\S 5 . \quad$ Several applications}

This section is divided into Sections 5.1-5.4. We apply the results obtained in Sections 3 and 4, respectively, to the Schrödinger operator or the degenerate Schrödinger operator on $\mathbb{R}^{d}$, and to the sub-Laplace Schrödinger operator on Heisenberg groups or on connected and simply connected nilpotent Lie groups.

\subsection{Schrödinger operators on $\mathbb{R}^{d}$}

Let $d \geq 3$, and let $\mathbb{R}^{d}$ be the $d$-dimensional Euclidean space endowed with the Euclidean norm $|\cdot|$ and the Lebesgue measure $d x$. Denote the Laplacian $\sum_{j=1}^{d} \frac{\partial^{2}}{\partial x_{j}^{2}}$ on $\mathbb{R}^{d}$ by $\Delta$ and the corresponding heat (Gauss) semigroup $\left\{e^{t \Delta}\right\}_{t>0}$ by $\left\{\widetilde{T}_{t}\right\}_{t>0}$. Let $V$ be a nonnegative locally integrable function on $\mathbb{R}^{d}$, let $\mathcal{L} \equiv-\Delta+V$ be the Schrödinger operator, and let $\left\{T_{t}\right\}_{t>0}$ be the corresponding semigroup with kernels $\left\{T_{t}(x, y)\right\}_{t>0}$. Moreover, for all $t>0$ and $x, y \in \mathbb{R}^{d}$, set

$$
\left.Q_{t}(x, y) \equiv t^{2} \frac{d T_{s}(x, y)}{d s}\right|_{s=t^{2}}
$$

Let $q \in(d / 2, d]$, let $V \in \mathcal{B}_{q}\left(\mathbb{R}^{d},|\cdot|, d x\right)$, and let $\rho$ be as in $(2.3)$. Then we have the following estimates (see [6], [9], [7]).

Proposition 5.1. Let $q \in(d / 2, d]$, let $\beta \in(0,2-d / q)$, and let $N \in \mathbb{N}$. Then there exist positive constants $\widetilde{C}$ and $C$, where $C$ is independent of $N$, such that for all $t \in(0, \infty)$ and $x, x^{\prime}, y \in \mathcal{X}$ with $d\left(x, x^{\prime}\right) \leq \sqrt{t} / 2$,

(i) $\left|T_{t}(x, y)\right| \leq \widetilde{C} t^{-d / 2} \exp \left\{-|x-y|^{2} / C t\right\}[\rho(x) /(\sqrt{t}+\rho(x))]^{N}[\rho(y) /$ $(\sqrt{t}+\rho(y))]^{N}$,

(ii) $\left|T_{t}(x, y)-T_{t}\left(x^{\prime}, y\right)\right| \leq \widetilde{C}\left[\left|x-x^{\prime}\right| / \sqrt{t}\right]^{\beta} t^{-d / 2} \exp \left\{-|x-y|^{2} / C t\right\}[\rho(x) /$ $(\sqrt{t}+\rho(x))]^{N}[\rho(y) /(\sqrt{t}+\rho(y))]^{N}$,

(iii) $\left|T_{t}(x, y)-\widetilde{T}_{t}(x, y)\right| \leq \widetilde{C}[\sqrt{t} /(\sqrt{t}+\rho(x))]^{2-d / q} t^{-d / 2} \exp \left\{-|x-y|^{2} /\right.$ $C t\}$;

and for all $t \in(0, \infty)$ and $x, x^{\prime}, y \in \mathcal{X}$ with $d\left(x, x^{\prime}\right) \leq t / 2$,

(iv) $\left|Q_{t}(x, y)\right| \leq \widetilde{C} t^{-d} \exp \left\{-|x-y|^{2} / C t^{2}\right\}[\rho(x) /(t+\rho(x))]^{N}[\rho(y) /(t+$ $\rho(y))]^{N}$, 
(v) $\left|Q_{t}(x, y)-Q_{t}\left(x^{\prime}, y\right)\right| \leq \widetilde{C}\left[\left|x-x^{\prime}\right| / t\right]^{\beta} t^{-d} \exp \left\{-|x-y|^{2} / C t^{2}\right\}[\rho(x) /$ $(t+\rho(x))]^{N}[\rho(y) /(t+\rho(y))]^{N}$,

(vi) $\left|\int_{\mathbb{R}}^{d} Q_{t}(x, y) d \mu(y)\right| \leq \widetilde{C}[t / \rho(x)]^{2-d / q}[\rho(x) /(t+\rho(x))]^{N}$.

Let $q_{1}, q_{2} \in(d / 2, \infty]$ with $q_{1}<q_{2}$. Observe that $\mathcal{B}_{q_{2}}\left(\mathbb{R}^{d}\right) \subset \mathcal{B}_{q_{1}}\left(\mathbb{R}^{d}\right)$. Therefore, Proposition 5.1 holds for all $q \in(d / 2, \infty]$. On the other hand, recall that $\left\{\widetilde{T}_{t^{2}}\right\}_{t>0}$ satisfies that for all $t \in(0, \infty), \widetilde{T}_{t^{2}}(1)=1$ (see [6], [9]). Thus $\left\{T_{t^{2}}\right\}_{t>0}$ satisfies assumptions (3.1)-(3.3). Moreover, the $L^{2}\left(\mathbb{R}^{d}\right)$-boundedness of $g$-function $g(\cdot)$ was obtained in [6]. Using this, Proposition 5.1(iv) and 5.1(v), and the vector-valued Calderón-Zygmund theory (see, e.g., [26]), we obtain the $L^{p}\left(\mathbb{R}^{d}\right)$-boundedness of $g(\cdot)$ for $p \in(1, \infty)$. Then by applying this fact and Proposition 5.1, Theorems 3.1, 3.2, and 4.1, and Corollary 4.1, we have the following result.

Proposition 5.2. Let $q \in(d / 2, \infty]$, let $p \in(1, \infty)$, let $V \in \mathcal{B}_{q}\left(\mathbb{R}^{d},|\cdot|, d x\right)$, and let $\rho$ be as in (2.3).

(i) If $\alpha \in(-\infty, 1 / d-1 /(2 q))$, then there exists a positive constant $C$ such that for all $f \in \mathcal{E}_{\rho}^{\alpha, p}\left(\mathbb{R}^{d}\right), T^{+}(f), P^{+}(f) \in \widetilde{\mathcal{E}}_{\rho}^{\alpha, p}\left(\mathbb{R}^{d}\right)$ and

$$
\left\|T^{+}(f)\right\|_{\tilde{\mathcal{E}}_{\rho}^{\alpha, p}\left(\mathbb{R}^{d}\right)}+\left\|P^{+}(f)\right\|_{\tilde{\mathcal{E}}_{\rho}^{\alpha, p}\left(\mathbb{R}^{d)}\right.} \leq C\|f\|_{\mathcal{E}_{\rho}^{\alpha, p}\left(\mathbb{R}^{d}\right)} .
$$

(ii) If $\alpha \in(-\infty, 2 /(3 d)-1 /(3 q))$, then there exists a positive constant $C$ such that for all $f \in \mathcal{E}_{\rho}^{\alpha, p}\left(\mathbb{R}^{d}\right),[g(f)]^{2} \in \widetilde{\mathcal{E}}_{\rho}^{2 \alpha, p / 2}\left(\mathbb{R}^{d}\right)$ with $\left\|[g(f)]^{2}\right\|_{\widetilde{\mathcal{E}}_{\rho}^{2 \alpha, p / 2}\left(\mathbb{R}^{d}\right)} \leq$ $C\|f\|_{\mathcal{E}_{\rho}^{\alpha, p}\left(\mathbb{R}^{d}\right)}^{2}$, and $g(f) \in \widetilde{\mathcal{E}}_{\rho}^{\alpha, p}\left(\mathbb{R}^{d}\right)$ with $\|g(f)\|_{\tilde{\mathcal{E}}_{\rho}^{\alpha, p}\left(\mathbb{R}^{d}\right)} \leq C\|f\|_{\mathcal{E}_{\rho}^{\alpha, p}\left(\mathbb{R}^{d}\right)}$.

\subsection{Degenerate Schrödinger operators on $\mathbb{R}^{d}$}

Let $d \geq 3$, and let $\mathbb{R}^{d}$ be the $d$-dimensional Euclidean space endowed with the Euclidean norm $|\cdot|$ and the Lebesgue measure $d x$. Recall that a nonnegative locally integrable function $w$ is said to be an $A_{2}\left(\mathbb{R}^{d}\right)$ weight in the sense of Muckenhoupt if

$$
\sup _{B \subset \mathbb{R}^{d}}\left\{\frac{1}{|B|} \int_{B} w(x) d x\right\}^{1 / 2}\left\{\frac{1}{|B|} \int_{B}[w(x)]^{-1} d x\right\}^{1 / 2}<\infty,
$$

where the supremum is taken over all the balls in $\mathbb{R}^{d}$. Observe that if we set $w(E) \equiv \int_{E} w(x) d x$ for any measurable set $E$, then there exist positive constants $C, Q$, and $\kappa$ such that for all $x \in \mathbb{R}^{d}, \lambda>1$, and $r>0$,

$$
C^{-1} \lambda^{\kappa} w(B(x, r)) \leq w(B(x, \lambda r)) \leq C \lambda^{Q} w(B(x, r))
$$


namely, the measure $w(x) d x$ satisfies $(2.1)$. Thus $\left(\mathbb{R}^{d},|\cdot|, w(x) d x\right)$ is a space of homogeneous type.

Let $w \in A_{2}\left(\mathbb{R}^{d}\right)$, and let $\left\{a_{i, j}\right\}_{1 \leq i, j \leq d}$ be a real symmetric matrix function satisfying that for all $x, \xi \in \mathbb{R}^{d}$,

$$
C^{-1}|\xi|^{2} \leq \sum_{1 \leq i, j \leq d} a_{i, j}(x) \xi_{i} \bar{\xi}_{j} \leq C|\xi|^{2} .
$$

Then the degenerate elliptic operator $\mathcal{L}_{0}$ is defined by

$$
\mathcal{L}_{0} f(x) \equiv-\frac{1}{w(x)} \sum_{1 \leq i, j \leq d} \partial_{i}\left(a_{i, j}(\cdot) \partial_{j} f\right)(x),
$$

where $x \in \mathbb{R}^{d}$. Denote by $\left\{\widetilde{T}_{t}\right\}_{t>0} \equiv\left\{e^{-t \mathcal{L}_{0}}\right\}_{t>0}$ the semigroup generated by $\mathcal{L}_{0}$.

Let $V$ be a nonnegative locally integrable function on $w(x) d x$. Define the degenerate Schrödinger operator by $\mathcal{L} \equiv \mathcal{L}_{0}+V$. Then $\mathcal{L}$ generates a semigroup $\left\{T_{t}\right\}_{t>0} \equiv\left\{e^{-t \mathcal{L}}\right\}_{t>0}$ with kernels $\left\{T_{t}(x, y)\right\}_{t>0}$. Moreover, for all $t \in(0, \infty)$ and $x, y \in \mathbb{R}^{d}$, set

$$
\left.Q_{t}(x, y) \equiv t^{2} \frac{d T_{s}(x, y)}{d s}\right|_{s=t^{2}} .
$$

Let $q \in(Q / 2, Q]$, let $V \in \mathcal{B}_{q}\left(\mathbb{R}^{d},|\cdot|, w(x) d x\right)$, and let $\rho$ be as in (2.3). Then $\left\{T_{t}(\cdot, \cdot)\right\}_{t>0}$ and $\left\{Q_{t}(\cdot, \cdot)\right\}_{t>0}$ satisfy Proposition 5.1 with $t^{-d / 2}$ replaced by $\left[V_{\sqrt{t}}(x)\right]^{-1}, t^{-d}$ by $\left[V_{t}(x)\right]^{-1}$ and $d$ by $Q$. In fact, the corresponding Proposition 5.1(i) and 5.1(iii) here were given in [6]. The proof of the corresponding Proposition 5.1(ii) here is similar to that of Proposition 5.1 (see [9]). The proofs of the corresponding Proposition 5.1(iv), 5.1(v), and 5.1(vi) here are similar to that of [7, Proposition 4]. We omit the details here.

Recall that $\left\{\widetilde{T}_{t^{2}}\right\}_{t>0}$ satisfies that for all $t \in(0, \infty), \widetilde{T}_{t^{2}}(1)=1$ (see, e.g., [13]). Thus $\left\{T_{t^{2}}\right\}_{t>0}$ satisfies assumptions (3.1)-(3.3). Moreover, the $L^{2}\left(\mathbb{R}^{d}\right)$ boundedness of $g(\cdot)$ can be obtained by the same argument as in $[6$, Lemma 3]. Using this, Proposition 5.1(iv) and 5.1(v), and the vector-valued Calderón-Zygmund theory, we obtain the $L^{p}\left(\mathbb{R}^{d}\right)$-boundedness of $g(\cdot)$ for $p \in(1, \infty)$. Then by applying these facts, Theorems $3.1,3.2$, and 4.1 , and Corollary 4.1, we have the following result.

Proposition 5.3. Let $w \in A_{2}\left(\mathbb{R}^{d}\right)$, let $q \in(Q / 2, \infty]$, let $p \in(1, \infty)$, let $V \in \mathcal{B}_{q}\left(\mathbb{R}^{d},|\cdot|, w(x) d x\right)$, and let $\rho$ be as in (2.3) with $d \mu(x)=w(x) d x$. 
(i) If $\alpha \in(-\infty, 1 / Q-1 /(2 q))$, then there exists a positive constant $C$ such that for all $f \in \mathcal{E}_{\rho}^{\alpha, p}(w(x) d x), T^{+}(f), P^{+}(f) \in \widetilde{\mathcal{E}}_{\rho}^{\alpha, p}(w(x) d x)$ and

$$
\left\|T^{+}(f)\right\|_{\widetilde{\mathcal{E}}_{\rho}^{\alpha, p}(w(x) d x)}+\left\|P^{+}(f)\right\|_{\widetilde{\mathcal{E}}_{\rho}^{\alpha, p}(w(x) d x)} \leq C\|f\|_{\mathcal{E}_{\rho}^{\alpha, p}(w(x) d x)} .
$$

(ii) If $\alpha \in(-\infty, 2 /(3 Q)-1 /(3 q))$, then there exists a positive constant $C$ such that for all $f \in \mathcal{E}_{\rho}^{\alpha, p}(w(x) d x), \quad[g(f)]^{2} \in \widetilde{\mathcal{E}}_{\rho}^{2 \alpha, p / 2}(w(x) d x)$ with $\left\|[g(f)]^{2}\right\|_{\widetilde{\mathcal{E}}_{\rho}^{2 \alpha, p / 2}(w(x) d x)} \leq C\|f\|_{\mathcal{E}_{\rho}^{\alpha, p}(w(x) d x)}^{2}$, and $g(f) \in \widetilde{\mathcal{E}}_{\rho}^{\alpha, p}(w(x) d x)$ with $\|g(f)\|_{\widetilde{\mathcal{E}}_{\rho}^{\alpha, p}(w(x) d x)} \leq C\|f\|_{\mathcal{E}_{\rho}^{\alpha, p}(w(x) d x)}$.

\subsection{Schrödinger operators on Heisenberg groups}

The $(2 n+1)$-dimensional Heisenberg group $\mathbb{H}^{n}$ is a connected and simply connected nilpotent Lie group with the underlying manifold $\mathbb{R}^{2 n} \times \mathbb{R}$ and the multiplication

$$
(x, s)(y, s)=\left(x+y, t+s+2 \sum_{j=1}^{n}\left[x_{n+j} y_{j}-x_{j} y_{n+j}\right]\right) .
$$

The homogeneous norm on $\mathbb{H}^{n}$ is defined by $|(x, t)|=\left(|x|^{4}+|t|^{2}\right)^{1 / 4}$ for all $(x, t) \in \mathbb{H}^{n}$, which induces a left-invariant metric $d((x, t),(y, s))=$ $|(-x,-t)(y, s)|$. Moreover, there exists a positive constant $C$ such that $|B((x, t), r)|=C r^{Q}$, where $Q=2 n+2$ is the homogeneous dimension of $\mathbb{H}^{n}$ and $|B((x, t), r)|$ is the Lebesgue measure of the ball $B((x, t), r)$. The triplet $\left(\mathbb{H}^{n}, d, d x\right)$ is a space of homogeneous type.

A basis for the Lie algebra of left invariant vector fields on $\mathbb{H}^{n}$ is given by

$$
\begin{aligned}
X_{2 n+1} & =\frac{\partial}{\partial t}, \quad X_{j}=\frac{\partial}{\partial x_{j}}+2 x_{n+j} \frac{\partial}{\partial t} \\
X_{n+j} & =\frac{\partial}{\partial x_{n+j}}-2 x_{j} \frac{\partial}{\partial t}, \quad j=1, \ldots, n .
\end{aligned}
$$

All nontrivial commutators are $\left[X_{j}, X_{n+j}\right]=-4 X_{2 n+1}, j=1, \ldots, n$. The sub-Laplacian has the form $\Delta_{\mathbb{H}^{n}}=\sum_{j=1}^{2 n} X_{j}^{2}$.

Let $V$ be a nonnegative locally integrable function on $\mathbb{H}^{n}$. Define the subLaplacian Schrödinger operator by $\mathcal{L} \equiv-\Delta_{\mathbb{H} n}+V$. Denote by $\left\{T_{t}\right\}_{t>0} \equiv$ $\left\{e^{-t \mathcal{L}}\right\}_{t>0}$ with kernels $\left\{T_{t}(x, y)\right\}_{t>0}$ and by $\left\{\widetilde{T}_{t}\right\}_{t>0} \equiv\left\{e^{t \Delta_{\mathbb{H}^{n}}}\right\}_{t>0}$. Moreover, for all $t \in(0, \infty)$ and $x, y \in \mathbb{R}^{d}$, set

$$
\left.Q_{t}(x, y) \equiv t^{2} \frac{d T_{s}(x, y)}{d s}\right|_{s=t^{2}} .
$$


Let $V \in \mathcal{B}_{q}\left(\mathbb{H}^{n}, d, d x\right)$ with $q \in(n+1,2 n+2]$, and let $\rho$ be as in (2.3). Then $\left\{T_{t}(\cdot, \cdot)\right\}_{t>0}$ and $\left\{Q_{t}(\cdot, \cdot)\right\}_{t>0}$ satisfy Proposition 5.1 with $d$ replaced by $2(n+2)$ and $|x-y|$ replaced by $d(x, y)$ (see [19]).

Observe that $\left\{\widetilde{T}_{t^{2}}\right\}_{t>0}$ satisfies that for all $t \in(0, \infty), \widetilde{T}_{t^{2}}(1)=1$ (see also $[32])$. Thus $\left\{T_{t^{2}}\right\}_{t>0}$ satisfies assumptions (3.1)-(3.3). Moreover, the $L^{2}\left(\mathbb{H}^{n}\right)$ boundedness of $g(\cdot)$ was obtained in [19]. Using this, Proposition 5.1(iv) and 5.1(v), and the vector-valued Calderón-Zygmund theory, we obtain the $L^{p}\left(\mathbb{H}^{n}\right)$-boundedness of $g(\cdot)$ for $p \in(1, \infty)$. Then by applying these facts, Theorems 3.1, 3.2, and 4.1, and Corollary 4.1, we have the following conclusions.

Proposition 5.4. Let $q \in(n+1, \infty]$, let $p \in(1, \infty)$, let $V \in \mathcal{B}_{q}\left(\mathbb{H}^{n}, d, d x\right)$, and let $\rho$ be as in (2.3).

(i) If $\alpha \in(-\infty, 1 /(2 n+2)-1 /(2 q))$, then there exists a positive constant $C$ such that for all $f \in \mathcal{E}_{\rho}^{\alpha, p}\left(\mathbb{H}^{n}\right), T^{+}(f), P^{+}(f) \in \widetilde{\mathcal{E}}_{\rho}^{\alpha, p}\left(\mathbb{H}^{n}\right)$ and

$$
\left\|T^{+}(f)\right\|_{\widetilde{\mathcal{E}}_{\rho}^{\alpha, p}\left(\mathbb{H}^{n}\right)}+\left\|P^{+}(f)\right\|_{\widetilde{\mathcal{E}}_{\rho}^{\alpha, p}\left(\mathbb{H}^{n}\right)} \leq C\|f\|_{\mathcal{E}_{\rho}^{\alpha, p}\left(\mathbb{H}^{n}\right)} .
$$

(ii) If $\alpha \in(-\infty, 1 /(3 n+3)-1 /(3 q))$, then there exists a positive constant $C$ such that for all $f \in \mathcal{E}_{\rho}^{\alpha, p}\left(\mathbb{H}^{n}\right),[g(f)]^{2} \in \widetilde{\mathcal{E}}_{\rho}^{2 \alpha, p / 2}\left(\mathbb{H}^{n}\right)$ with $\left\|[g(f)]^{2}\right\|_{\widetilde{\mathcal{E}}_{\rho}^{2 \alpha, p / 2}\left(\mathbb{H}^{n}\right)}$ $\leq C\|f\|_{\mathcal{E}_{\rho}^{\alpha, p}\left(\mathbb{H}^{n}\right)}^{2}$, and $g(f) \in \widetilde{\mathcal{E}}_{\rho}^{\alpha, p}\left(\mathbb{H}^{n}\right)$ with $\|g(f)\|_{\widetilde{\mathcal{E}}_{\rho}^{\alpha, p}\left(\mathbb{H}^{n}\right)} \leq C\|f\|_{\mathcal{E}_{\rho}^{\alpha, p}\left(\mathbb{H}^{n}\right)}$.

\subsection{Schrödinger operators on connected and simply connected nilpotent Lie groups}

Let $\mathbb{G}$ be a connected and simply connected nilpotent Lie group, and let $X \equiv\left\{X_{1}, \ldots, X_{k}\right\}$ be left-invariant vector fields on $\mathbb{G}$ satisfying the Hörmander condition that $\left\{X_{1}, \ldots, X_{k}\right\}$ together with their commutators of order $\leq m$ generates the tangent space of $\mathbb{G}$ at each point of $\mathbb{G}$. Let $d$ be the Carnot-Carathéodory (control) distance on $\mathbb{G}$ associated to $\left\{X_{1}, \ldots, X_{k}\right\}$. Fix a left-invariant Haar measure $\mu$ on $\mathbb{G}$. Then for all $x \in \mathbb{G}, V_{r}(x)=V_{r}(e)$; moreover, there exist $\kappa, D \in(0, \infty)$ with $\kappa \leq D$ such that for all $x \in \mathbb{G}$, $C^{-1} r^{\kappa} \leq V_{r}(x) \leq C r^{\kappa}$ when $r \in(0,1]$, and $C^{-1} r^{D} \leq V_{r}(x) \leq C r^{D}$ when $r \in(1, \infty)$ (see [21], [30], [31]). Thus $(\mathbb{G}, d, \mu)$ is a space of homogeneous type.

The sub-Laplacian is given by $\Delta_{\mathbb{G}} \equiv \sum_{j=1}^{k} X_{j}^{2}$. Denote by $\left\{\widetilde{T}_{t}\right\}_{t>0} \equiv$ $\left\{e^{t \Delta_{\mathbb{G}}}\right\}_{t>0}$ the semigroup generated by $-\Delta_{\mathbb{G}}$.

Let $V$ be a nonnegative locally integrable function on $\mathbb{G}$. Then the subLaplace Schrödinger operator $\mathcal{L}$ is defined by $\mathcal{L} \equiv-\Delta_{\mathbb{G}}+V$. The operator $\mathcal{L}$ generates a semigroup of operators $\left\{T_{t}\right\}_{t>0} \equiv\left\{e^{-t \mathcal{L}}\right\}_{t>0}$, whose kernels 
are denoted by $\left\{T_{t}(x, y)\right\}_{t>0}$. Define the radial maximal operator $T^{+}$by $T^{+}(f)(x) \equiv \sup _{t>0}\left|e^{-t \mathcal{L}}(f)(x)\right|$ for all $x \in \mathbb{G}$.

Let $q>D / 2$, let $V \in \mathcal{B}_{q}(\mathbb{G}, d, \mu)$, and let $\rho$ be as in $(2.3)$. For all $x, y \in \mathbb{G}$ and $t \in(0, \infty)$, define

$$
\left.Q_{t}(x, y) \equiv t^{2} \frac{d}{d s}\right|_{s=t^{2}} T_{s}(x, y) .
$$

Then $\left\{T_{t}(\cdot, \cdot)\right\}_{t>0}$ and $\left\{Q_{t}(\cdot, \cdot)\right\}_{t>0}$ satisfy Proposition 5.1 with $t^{-d}$ replaced by $\left[V_{t}(x)\right]^{-1}, t^{-d / 2}$ by $\left[V_{\sqrt{t}}(x)\right]^{-1}$, and $d$ by $D$ (see [33], [32]). Observe that $\left\{\widetilde{T}_{t^{2}}\right\}_{t>0}$ satisfies that for all $t \in(0, \infty), \widetilde{T}_{t^{2}}(1)=1$ (see, e.g., [30]). Thus $\left\{T_{t^{2}}\right\}_{t>0}$ satisfies assumptions (3.1)-(3.3). Moreover, the $L^{2}(\mathbb{G})$-boundedness of $g(\cdot)$ can be obtained by the same argument as in [6, Lemma 3]. Using this, Proposition 5.4(iv) and 5.4(v), and the vector-valued Calderón-Zygmund theory, we obtain the $L^{p}(\mathbb{G})$-boundedness of $g(\cdot)$ for $p \in(1, \infty)$. Then by applying these facts, Theorems 3.1, 3.2, and 4.1, and Corollary 4.1, we have the following conclusions.

Proposition 5.5. Let $q \in(D / 2, \infty]$, let $p \in(1, \infty)$, let $V \in \mathcal{B}_{q}(\mathbb{G}, d, \mu)$, and let $\rho$ be as in (2.3).

(i) If $\alpha \in(-\infty, 1 / D-1 /(2 q))$, then there exists a positive constant $C$ such that for all $f \in \mathcal{E}_{\rho}^{\alpha, p}(\mathbb{G}), T^{+}(f), P^{+}(f) \in \widetilde{\mathcal{E}}_{\rho}^{\alpha, p}(\mathbb{G})$ and

$$
\left\|T^{+}(f)\right\|_{\widetilde{\mathcal{E}}_{\rho}^{\alpha, p}(\mathbb{G})}+\left\|P^{+}(f)\right\|_{\widetilde{\mathcal{E}}_{\rho}^{\alpha, p}(\mathbb{G})} \leq C\|f\|_{\mathcal{E}_{\rho}^{\alpha, p}(\mathbb{G})} .
$$

(ii) If $\alpha \in(-\infty, 2 /(3 D)-1 /(3 q))$, then there exists a positive constant $C$ such that for all $f \in \mathcal{E}_{\rho}^{\alpha, p}(\mathbb{G}),[g(f)]^{2} \in \widetilde{\mathcal{E}}_{\rho}^{2 \alpha, p / 2}(\mathbb{G})$ with $\left\|[g(f)]^{2}\right\|_{\widetilde{\mathcal{E}}_{\rho}^{2 \alpha, p / 2}(\mathbb{G})} \leq$ $C\|f\|_{\mathcal{E}_{\rho}^{\alpha, p}(\mathbb{G})}^{2}$, and $g(f) \in \widetilde{\mathcal{E}}_{\rho}^{\alpha, p}(\mathbb{G})$ with $\|g(f)\|_{\widetilde{\mathcal{E}}_{\rho}^{\alpha, p}(\mathbb{G})} \leq C\|f\|_{\mathcal{E}_{\rho}^{\alpha, p}(\mathbb{G})}$.

\section{REFERENCES}

[1] S. Campanato, Proprietà di hölderianità di alcune classi di funzioni, Ann. Sc. Norm. Super. Pisa 17 (1963), 175-188.

[2] R. R. Coifman and R. Rochberg, Another characterization of BMO, Proc. Amer. Math. Soc. 79 (1980), 249-254.

[3] R. R. Coifman and G. Weiss, Analyse Harmonique Non-commutative sur Certains Espaces Homogènes, Lecture Notes in Math. 242, Springer, Berlin, 1971.

[4] R. R. Coifman and G. Weiss, Extensions of Hardy spaces and their use in analysis, Bull. Amer. Math. Soc. 83 (1977), 569-645.

[5] X. T. Duong, J. Xiao, and L. Yan, Old and new Morrey spaces with heat kernel bounds, J. Fourier Anal. Appl. 13 (2007), 87-111. 
[6] J. Dziubański, Note on $H^{1}$ spaces related to degenerate Schrödinger operators, Illinois J. Math. 49 (2005), 1271-1297.

[7] J. Dziubański, G. Garrigós, T. Martínez, J. L. Torrea, and J. Zienkiewicz, BMO spaces related to Schrödinger operators with potentials satisfying a reverse Hölder inequality, Math. Z. 249 (2005), 329-356.

[8] J. Dziubański and J. Zienkiewicz, Hardy space $H^{1}$ associated to Schrödinger operator with potential satisfying reverse Hölder inequality, Rev. Mat. Iberoam. 15 (1999), 279-296.

[9] J. Dziubański and J. Zienkiewicz, $H^{p}$ spaces associated with Schrödinger operators with potentials from reverse Hölder classes, Colloq. Math. 98 (2003), 5-38.

[10] C. Fefferman, The uncertainty principle, Bull. Amer. Math. Soc. (N.S.) 9 (1983), 129-206.

[11] D. Goldberg, A local version of real Hardy spaces, Duke Math. J. 46 (1979), 27-42.

[12] Y. Han, D. Müller, and D. Yang, A theory of Besov and Triebel-Lizorkin spaces on metric measure spaces modeled on Carnot-Carathéodory spaces, Abstr. Appl. Anal. 2008, no. 893409.

[13] W. Hebisch and L. Saloff-Coste, On the relation between elliptic and parabolic Harnack inequalities, Ann. Inst. Fourier (Grenoble) 51 (2001), 1437-1481.

[14] G. Hu, Y. Meng, and D. Yang, Estimates for Marcinkiewicz integrals in BMO and Campanato spaces, Glasg. Math. J. 49 (2007), 167-187.

[15] G. Hu, D. Yang, and D. Yang, $h^{1}$, bmo, blo and Littlewood-Paley g-functions with non-doubling measures, Rev. Mat. Iberoam. 25 (2009), 595-667.

[16] J. Huang and H. Liu, Area integrals associated to Schrödinger operators, preprint.

[17] P. G. Lemarié-Rieusset, The Navier-Stokes equations in the critical MorreyCampanato space, Rev. Mat. Iberoam. 23 (2007), 897-930.

[18] H. Li, Estimations $L^{p}$ des opérateurs de Schrödinger sur les groupes nilpotents, J. Funct. Anal. 161 (1999), 152-218.

[19] C. Lin and H. Liu, The BMO-type space $B M O_{\mathcal{L}}$ associated with Schrödinger operators on the Heisenberg group, preprint.

[20] R. A. Macías and C. Segovia, Lipschitz functions on spaces of homogeneous type, Adv. Math. 33 (1979), 257-270.

[21] A. Nagel, E. M. Stein, and S. Wainger, Balls and metrics defined by vector fields I. Basic properties, Acta Math. 155 (1985), 103-147.

[22] E. Nakai, The Campanato, Morrey and Hölder spaces on spaces of homogeneous type, Studia Math. 176 (2006), 1-19.

[23] E. Nakai, Orlicz-Morrey spaces and the Hardy-Littlewood maximal function, Studia Math. 188 (2008), 193-221.

[24] J. Peetre, On the theory of $\mathcal{L}_{p, \lambda}$ spaces, J. Funct. Anal. 4 (1969), 71-87.

[25] Z. Shen, $L^{p}$ estimates for Schrödinger operators with certain potentials, Ann. Inst. Fourier (Grenoble) 45 (1995), 513-546.

[26] E. M. Stein, Harmonic Analysis: Real-Variable Methods, Orthogonality, and Oscillatory Integrals, Princeton University Press, Princeton, 1993.

[27] J.-O. Strömberg and A. Torchinsky, Weighted Hardy Spaces, Lecture Notes in Math. 1381, Springer, Berlin, 1989.

[28] M. H. Taibleson and G. Weiss, "The molecular characterization of certain Hardy spaces," in Representation Theorems for Hardy Spaces, Astérisque 77, Soc. Math. France, Paris, 1980, 67-149.

[29] H. Triebel, Theory of Function Spaces, Vol. II, Birkhäuser, Basel, 1992. 
[30] N. T. Varopoulos, Analysis on Lie groups, J. Funct. Anal. 76 (1988), 346-410.

[31] N. T. Varopoulos, L. Saloff-Coste, and T. Coulhon, Analysis and Geometry on Groups, Cambridge University Press, Cambridge, 1992.

[32] D. Yang, D. Yang, and Y. Zhou, Localized BMO and BLO spaces on RD-spaces and applications to Schrödinger operators, Commun. Pure Appl. Anal. 9 (2010), 779-812.

[33] D. Yang and Y. Zhou, Localized Hardy spaces $H^{1}$ related to admissible functions on RD-spaces and applications to Schrödinger operators, to appear in Trans. Amer. Math. Soc.

[34] J. Zhong, The Sobolev estimates for some Schrödinger type operators, Math. Sci. Res. Hot-Line 3 (1999), 1-48.

Dachun Yang

School of Mathematical Sciences

Beijing Normal University

Laboratory of Mathematics and Complex Systems

Ministry of Education

Beijing 100875

People's Republic of China

dcyang@bnu.edu.cn

Dongyong Yang

School of Mathematical Sciences

Beijing Normal University

Laboratory of Mathematics and Complex Systems

Ministry of Education

Beijing 100875

People's Republic of China

dyyang@mail. bnu.edu.cn

Yuan Zhou

School of Mathematical Sciences

Beijing Normal University

Laboratory of Mathematics and Complex Systems

Ministry of Education

Beijing 100875

People's Republic of China

yuanzhou@mail. bnu.edu.cn 